\title{
Exotic Quantum Criticality in One-dimensional Coupled Dipolar Bosons Tubes
}

\author{
P. Lecheminant ${ }^{1}$ and H. Nonne ${ }^{2}$ \\ ${ }^{1}$ Laboratoire de Physique Théorique et Modélisation, CNRS UMR 8089, \\ Université de Cergy-Pontoise, Site de Saint-Martin, F-95300 Cergy-Pontoise Cedex, France \\ ${ }^{2}$ Department of Physics, Technion, Haifa 32000, Israel
}

(Dated: March 24, 2021)

\begin{abstract}
The competition between intertube hopping processes and density-density interactions is investigated in onedimensional quantum dipolar bosons systems of $N$ coupled tubes at zero temperature. Using a phenomenological bosonization approach, we show that the resulting competition leads to an exotic quantum phase transition described by a $\mathrm{U}(1) \times \mathbb{Z}_{N}$ conformal field theory with a fractional central charge. The emerging $\mathbb{Z}_{N}$ parafermionic critical degrees of freedom are highly nontrivial in terms of the original atoms or polar molecules of the model. We further determine the main physical properties of the quantum critical point in a double-tube system which has central charge $c=3 / 2$. In triple-tube systems, we show that the competition between the two antagonistic processes is related to the physics of the two-dimensional $\mathbb{Z}_{3}$ chiral Potts model. This work opens the possibility to study the exotic properties of the $\mathbb{Z}_{N}$ parafermions in the context of ultracold quantum Bose gases.
\end{abstract}

PACS numbers: 71.10.Pm, 05.30.Jp

\section{INTRODUCTION}

Ultracold atomic systems have become one of the most experimentally flexible systems to study strongly correlated physics. The possibility of tuning the contact inter-particle interactions by varying the scattering length through Feshbach resonances has allowed the observation of many interesting phenomena like the BEC-BCS crossover in atomic Fermi gas. 1

On top of this wide tunability of the interaction strength, the possibility of controlling the shape of interactions through the long-range and anisotropic nature of dipole-dipole interactions has recently attracted considerable attention. In particular, dipolar interactions with long-range anisotropic character have been observed in chromium atoms by exploiting its large magnetic moments. ${ }^{2}$ An alternative route is to consider heteronuclear polar molecules with large electric dipole moments associated with their rotational excitations. ${ }^{3 / 4}$ The resulting long-range interactions between such polar molecules can be tuned using dc and ac electric fields $\frac{566}{{ }^{5}}$ Dipole-dipole interactions can then be much stronger than the superexchange interactions between ultracold atoms. This opens an avenue to realize a plethora of interesting quantum phases governed by long-range interactions. On the one hand, a variety of exotic phases have already been predicted to occur in dipolar quantum gases such as topological p-wave fermionic superfluids 7 and quantum nematic fluids $\stackrel{89}{89}$ On the other hand, as shown recently in Ref 10 , ultracold polar molecules become promising candidates for the simulation of condensed matter phenomena and could provide a robust toolbox for quantum information processing.

In this paper, we investigate the emergence of an exotic quantum criticality in coupled one-dimensional (1D) quantum bosons tubes with dipole-dipole interactions. The standard quantum critical behavior of 1D quantum bosons is the well-known Luttinger universality class 11 Its low-energy properties are governed by a relativistic free massless boson field $\Phi$ with a sound velocity $v$ and a Luttinger param- eter $K$ which depend on the density and interactions of the underlying 1D quantum Bose gas. A hallmark of the Luttinger phenomenology is the power-law decay of the physical quantities with non-universal exponents related to the Luttinger parameter $K$. Such universality class can in turn be viewed as a conformal field theory (CFT) with U(1) global continuous symmetry and central charge $c=1$ associated to the gapless free-bosonic mode $\Phi !^{15[16}$ A more complicate quantum-critical behavior has been found recently with ultracold bosons with the possible realization of the 2D Ising universality class ${ }^{17 . \mid 21}$ In that case, the criticality emerges at a quantum critical point characterized by a $\mathbb{Z}_{2}$ CFT with central charge $c=1 / 2$. The resulting gapless degree of freedom is a free massless Majorana (real) fermion which is half of a Dirac (complex) fermion. ${ }^{15 / 16}$ Here, in the context of coupled 1D dipolar bosons, we analyse the stabilization of exotic quantum critical points which are described by the $\mathrm{SU}(2)_{N}$ CFT with central charge $c=3 N /(N+2){ }^{[22}$ Equivalently, the emergent quantum criticality can be captured by the product of a conventional U(1) CFT with the $\mathbb{Z}_{N}$ parafermions CFT, with central charge $c=2(N-1) /(N+2)$, which is known to govern the critical properties of the $2 \mathrm{D} \mathbb{Z}_{N}$ generalization of Ising models. ${ }^{2324}$ Such nontrivial quantum criticality for $N>2$ cannot be described by a simple free-field theory in terms of 1D gapless bosons or fermions as it is the case for the Luttinger and Ising universality classes. In this respect, the resulting critical degrees of freedom are highly nonlocal with respect to the original atoms or molecules. Recently, the $\mathbb{Z}_{N}$ parafermions CFT has attracted a lot of interest in the context of non-Abelian fractional quantum Hall states. The so-called Read-Rezayi states are described in terms of the $\mathbb{Z}_{N}$ CFT and their excitations display non-Abelian statistics which stem from the parafermionic behavior of these states. ${ }^{25}$ Our work opens the possible realization of this exotic CFT with fractional central charge in the context of ultracold atoms or polar molecules.

The experimental setup that allows to create a system of coupled 1D dipolar bosons tubes, is similar to the one de- 


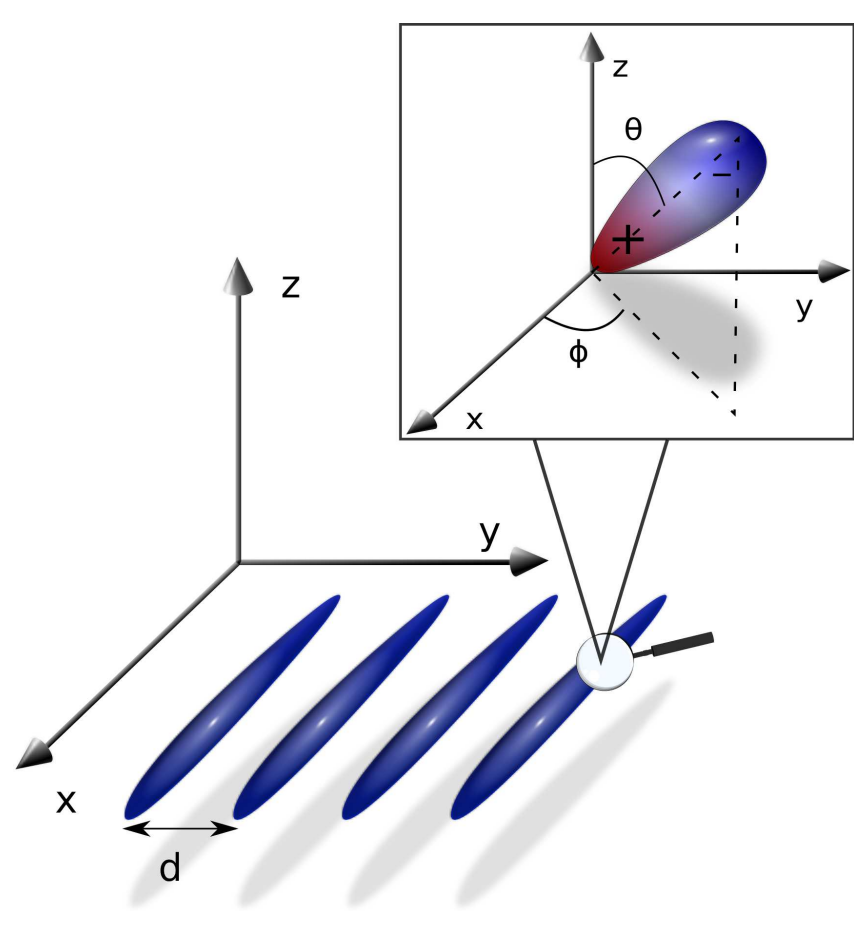

FIG. 1: (color online) Planar array of 1D tubes of dipolar boson gas positioned along the $x$ axis. The dipoles are all aligned along an additional external field.

scribed in Refs 26-31. A planar array of 1D tubes of dipolar boson gas, as shown in Fig. 11 is formed by an anisotropic 2D optical lattice in the $z-y$ plane. The strong confinement in the $z$-direction completely suppresses the hopping in that direction, while we allow a weak hopping between the tubes along the $y$-direction. Neglecting the inhomogeneity of the trapping potential, the system is thus a stack of $2 \mathrm{D}$ arrays, containing identical (straight) tubes of bosons, all oriented along the $x$-axis and separated by a distance $d=\lambda / 2$ in the $y$-direction ( $\lambda$ being the wavelength of the laser). The dipole moments of the bosons are aligned by an additional external field which is electric in the case of polar molecules or magnetic for chromium atoms. The dipole-dipole interaction strength can be tuned by using a rotating field, which induces a precession of the dipoles around the $z$-axis. ${ }^{[2] 32}$

The resulting effective Hamiltonian for this system can be written as:

$$
\mathcal{H}=\mathcal{H}_{\text {intra }}+\mathcal{H}_{\text {inter }}
$$

$\mathcal{H}_{\text {intra }}$ is the Hamiltonian describing the bosons' motion and interactions within the same tube:

$$
\begin{aligned}
& \mathcal{H}_{\text {intra }}=\sum_{n=1}^{N} \int d x \Psi_{n}^{\dagger}(x)\left(-\frac{\hbar^{2}}{2 M} \frac{d^{2}}{d x^{2}}\right) \Psi_{n}(x) \\
& +\sum_{n=1}^{N} \int d x_{1} d x_{2} V_{d d}^{\text {intra }}\left(x_{1}-x_{2}\right) \rho_{n}\left(x_{1}\right) \rho_{n}\left(x_{2}\right),(2)
\end{aligned}
$$

where $\Psi_{n}^{\dagger}(x)$ is the bosonic operator that creates a dipole at position $x$ in tube $n ; \rho_{n}(x)=\Psi_{n}^{\dagger}(x) \Psi_{n}(x)$ is the density operator and normal ordering is implicit throughout this paper. In Eq. 2), $M$ is the mass of the bosons and $V_{d d}^{\text {intra }}$ denotes the intratube dipole-dipole interactions. The coupling between the tubes is composed of the hopping along the $y$ direction, with amplitude $J_{\perp}$, and the intertube dipole-dipole interactions described by $V_{d d}^{\text {inter. }}$ :

$$
\begin{aligned}
& \mathcal{H}_{\text {inter }}=-J_{\perp} \sum_{n=1}^{N-1} \int d x\left[\Psi_{n}^{\dagger}(x) \Psi_{n+1}(x)+H . c .\right] \\
& +\sum_{n=1}^{N-1} \int d x_{1} d x_{2} V_{d d}^{\text {inter }}\left(x_{1}-x_{2}\right) \rho_{n}\left(x_{1}\right) \rho_{n+1}\left(x_{2}\right) .
\end{aligned}
$$

Here, we only consider coupling between nearest-neighbor tubes as in Ref 26. Unless stated otherwise (in Sec. IV), open-boundary conditions in the transverse direction $(y)$ are also assumed in Eq. (3). The two terms in Eq. (3) are of very different nature and can be tuned independently: the depth of the optical lattice potential in the $y$ direction controls the intertube hopping amplitude $J_{\perp}$, while $V_{d d}^{\text {inter }}$ is controlled by the rotating polarizing field.

In this paper, we will investigate the competition between these two sources of coupling, at zero temperature, and in the $1 \mathrm{D}$ regime where the total length in the transverse direction, i.e. $N d$, is finite. In this respect, small values of $N$, i.e. $N=2$ or 3 , can be obtained in principle, by selective evaporation of the tubes by using electric or magnetic field gradients. Alternatively, double-tube system can also be engineered by considering double-well optical lattices. ${ }^{[3}$ We will show that the competition between hopping processes and density-density interactions of Eq. (3) strongly depends on the value of the Luttinger parameter $\mathrm{K}$ of the $1 \mathrm{D}$ tubes. For bosons with contact interactions, $\mathrm{K}$ is larger than one and the hopping term turns out to be the leading contribution. ${ }^{34}$ A transition occurs, in the Berezinsky-Kosterlitz-Thouless (BKT) universality class, between the Luttinger phase where the $N$ tubes are decoupled and a superfluid phase with phase-coherence between neighboring tubes ${ }^{34}$ In stark contrast to contact interactions, we will show that a different scenario happens for 1D dipolar quantum bosons, which can have $K<1$ due to the non-local nature of the dipole-dipole interactions. ${ }^{[35}$ In the regime $K<1$, the hopping and density-density interactions strongly compete and a quantum phase transition takes place between a superfluid phase and a density or charge-density wave (CDW). The resulting transition becomes exotic and is described by a $U(1) \times \mathbb{Z}_{N}$ conformal field theory with central charge $c=3 N /(N+2)$.

The rest of the paper is organized as follows. In Sec. II, we follow a low-energy approach for the model (1) in the general $N$ case, using the phenomenological bosonization of the bosons $\frac{11-14}{1-1}$ supplemented by CFT techniques. There, we reveal the existence of a quantum critical point in the $\mathrm{SU}(2)_{N}$ universality class, which describes the competition between hopping and intertube interactions. The properties of this fixed point and its stability with respect to general perturbations are further analysed in the special cases $N=2,3$ in Sec. III and IV. In particular, we show that the low-energy properties of triple-tube systems are related to the physics of the $\mathbb{Z}_{3}$ 
chiral Potts model in the vicinity of the three-state Potts critical point ${ }^{36}$ Finally, Sec. V contains our concluding remarks. The paper is supplied with an Appendix which provides some important technical details.

\section{QUANTUM PHASE TRANSITION IN THE GENERAL $N$ TUBE CASE}

In this section, we will investigate the nature of the quantum phase transition which stems from the competition between the hopping term and the intertube density-density interaction of Eq. (3) in the general $N$ case.

\section{A. Phenomenological bosonization approach}

Let us first specify the form of the dipole-dipole interactions which appear in Eqs. (2, 3). The dipole moments are polarized along a direction $\mathbf{d}=(\sin \theta \cos \phi, \sin \theta \sin \phi, \cos \theta)$ in the spherical coordinates (see Fig. 1). The energy due to the interaction between two dipoles at relative position $\mathbf{r}$ is:

$$
V_{\mathrm{dd}}(\mathbf{r})=\frac{C_{d d}}{4 \pi} \frac{r^{2}-3(\mathbf{d} \cdot \mathbf{r})^{2}}{r^{5}},
$$

where the coupling constant is $C_{d d}=p^{2} / \epsilon_{0}$ for electric dipoles of strength $p$, and $C_{d d}=\mu_{0} \mu^{2}$ for magnetic dipoles of strength $\mu ; \epsilon_{0}$ and $\mu_{0}$ are respectively the vacuum permittivity and permeability. For two dipoles in the same tube, the dipole-dipole interaction is then:

$$
V_{\mathrm{dd}}^{\text {intra }}\left(x_{1}-x_{2}\right)=\frac{C_{d d}}{4 \pi} \frac{1-3 \sin ^{2} \theta \cos ^{2} \phi}{\left|x_{1}-x_{2}\right|^{3}} .
$$

A crucial hypothesis in our work is that the dipole-dipole interaction along the tubes should be repulsive to reach the regime $K<1$. A standard choice to get repulsive interactions along and between the tubes is to fix $\theta$ to its magic angle value: $\sin \theta=1 / \sqrt{3}$ as in $\operatorname{Ref} 26$ for instance. However, as we will show thereafter, repulsive intertube dipole-dipole interactions are not crucial for the existence of the quantum phase transition we investigate. Without loss of generality regarding the nature of the intertube interactions, we will consider a simpler choice $\theta=\phi=\pi / 2$, where the dipoles are polarized along the $y$-axis, perpendicular to the tubes axis. We thus get a repulsive intratube interaction:

$$
V_{\mathrm{dd}}^{\text {intra }}\left(x_{1}-x_{2}\right)=\frac{C_{d d}}{4 \pi} \frac{1}{\left|x_{1}-x_{2}\right|^{3}} .
$$

As in $\operatorname{Ref} 26$, we approximate the dipole-dipole interaction between two nearest-neighbor tubes by its leading contribution:

$$
V_{\mathrm{dd}}^{\text {inter }}\left(x_{1}-x_{2}\right) \simeq-\frac{C_{d d}}{2 \pi d^{3}} \delta\left(x_{1}-x_{2}\right),
$$

so that we then have a short-distance intertube attraction. The later approximation is very convenient to perform a fieldtheory analysis as in weakly coupled fermionic or bosonic ladders. ${ }^{12 \mid 14 / 16}$ Hence, we do not treat the full dipole-dipole interaction (4) in coupled tubes like in Ref 37, where the same system was considered, but without intertube hopping. However, the dipole interaction in quasi-1D (or in 2D) is not fully long-ranged as in $3 \mathrm{D}$, in the sense that its volume integral does not diverge with the system size. The crude approximation (7) might thus be enough to shed light on the competition between superfluidity and the CDW ordering in coupled tubes. In this respect, we will find that model (1) displays a quantum-critical point and we will investigate its stability under generic perturbations - perturbations that include in particular the longer range terms, neglected in $V_{\mathrm{dd}}^{\text {inter }}$.

With these specifications, we are now in position to study the physical properties of model (1) by means of the phenomenological bosonization (or harmonic-fluid approach). It is a powerful method that allows to study the lowenergy properties of strongly interacting 1D quantum bosons systems. ${ }^{11}$ The density operators $\rho_{n}(x), n=1, \ldots, N$ can be expressed in terms of $N$ bosonic quantum fields $\varphi_{n}(x)$ through! 14

$\rho_{n}(x) \simeq\left[\rho_{0}+\frac{1}{\sqrt{\pi}} \partial_{x} \varphi_{n}(x)\right] \sum_{m=-\infty}^{\infty} \alpha_{m} e^{2 i m\left(\sqrt{\pi} \varphi_{n}(x)+\pi \rho_{0} x\right)}$,

where $\alpha_{m}$ are non-universal constants and $\rho_{0}$ is the average density in each tube around which the fluctuations take place. We introduce the dual bosons $\vartheta_{n}$ associated to the fields $\varphi_{n}$ such that $\left[\varphi_{n}\left(x_{1}\right), \partial_{x} \vartheta_{m}\left(x_{2}\right)\right]=i \delta_{n m} \delta\left(x_{1}-x_{2}\right)$. They allow us to describe the phase fluctuations of the boson creation operator $\Psi_{n}^{\dagger}$ :

$$
\begin{aligned}
\Psi_{n}^{\dagger}(x) \simeq & {\left[\rho_{0}+\frac{1}{\sqrt{\pi}} \partial_{x} \varphi_{n}(x)\right]^{1 / 2} \exp \left(i \sqrt{\pi} \vartheta_{n}(x)\right) } \\
& \times \sum_{m=-\infty}^{\infty} \beta_{m} e^{2 i m\left(\sqrt{\pi} \varphi_{n}(x)+\pi \rho_{0} x\right)},
\end{aligned}
$$

$\beta_{m}$ being non-universal constants. In practice, one often only needs the leading parts of the expansions $(899)$ :

$$
\begin{aligned}
\rho_{n}(x) \sim \rho_{0}+ & \frac{1}{\sqrt{\pi}} \partial_{x} \varphi_{n}(x) \\
& +2 \rho_{0} \cos \left(\sqrt{4 \pi} \varphi_{n}(x)+2 \pi \rho_{0} x\right) \\
\Psi_{n}^{\dagger}(x) \sim \sqrt{\rho_{0}} & \exp \left(i \sqrt{\pi} \vartheta_{n}(x)\right) .
\end{aligned}
$$

After investigation of the effects of these leading parts, the higher harmonics and less relevant terms can then be studied a posteriori.

In the absence of intertube coupling, the low-energy properties of the Hamiltonian (2) are described by the TomonagaLuttinger model $: 35$

$$
\mathcal{H}_{\text {intra }}=\sum_{n=1}^{N} \int d x \frac{v}{2}\left[K\left(\partial_{x} \varphi_{n}\right)^{2}+\frac{1}{K}\left(\partial_{x} \vartheta_{n}\right)^{2}\right]
$$

where the velocity $v$, and the Luttinger parameter $K$ depend on the microscopic details of the original model. The Luttinger parameter has been determined numerically as function 
of the dipole coupling constant $C_{d d}$ and the average density $\rho_{0} \cdot \frac{[30[35]}{}$ In sharp contrast to bosonic gases with contact interactions where $K \geq 1, K$ can be much smaller than 1 for dipolar interactions.

The leading-part of the intertube interaction (3) can be expressed in terms of the boson fields $\varphi_{n}$ and $\vartheta_{n}$ using Eq. (10):

$$
\begin{aligned}
\mathcal{H}_{\text {inter }} & =\int d x \sum_{n=1}^{N-1}\left[-\frac{g_{\perp}}{a^{2}} \cos \left(\sqrt{\pi}\left(\vartheta_{n+1}-\vartheta_{n}\right)\right)\right. \\
& \left.-\frac{g_{d}}{a^{2}} \cos \left(\sqrt{4 \pi}\left(\varphi_{n+1}-\varphi_{n}\right)\right)\right] \\
& +\lambda \int d x \sum_{n=1}^{N-1} \partial_{x} \varphi_{n+1} \partial_{x} \varphi_{n}
\end{aligned}
$$

$a \sim 1 / \rho_{0}$ being the short distance cut off (the average interparticle distance). For the attractive dipole-dipole interaction (7), the coupling constants read as follows :

$$
\begin{aligned}
g_{\perp} & =2 \rho_{0} a^{2} J_{\perp} \\
g_{d} & =\frac{C_{d d} \rho_{0}^{2} a^{2}}{\pi d^{3}} \\
\lambda & =-\frac{C_{d d}}{2 \pi^{2} d^{3}} .
\end{aligned}
$$

At this point, it is worth noting that the sign of the two cosine operators of Eq. (12) can be changed by the canonical transformation on the bosonic fields:

$$
\begin{aligned}
& \varphi_{2 n} \rightarrow \varphi_{2 n}+\frac{\sqrt{\pi}}{4}, \quad \varphi_{2 n+1} \rightarrow \varphi_{2 n+1} \\
& \vartheta_{2 n} \rightarrow \vartheta_{2 n}+\sqrt{\pi}, \quad \vartheta_{2 n+1} \rightarrow \vartheta_{2 n+1},
\end{aligned}
$$

so that the case of repulsive intertube interactions can be treated on the same footing by using the transformation (14).

Let us discuss the different terms that appear in the interacting Hamiltonian (12). They are of very different nature. The forward scattering term with the coupling constant $\lambda$ will renormalize the velocity and Luttinger parameter of the bosonic modes. Now, the structure of the remaining terms is intriguing. They describe the competition between the bosonic fields $\varphi_{n}$ and their dual fields $\vartheta_{n}$. The low-energy effective Hamiltonian (12) is, in fact, similar to the competition between superconductivity and CDW in spin-gap electronic ladder systems, $\stackrel{38 \mid 39}{ }$ the two first terms in Eq. 12 being respectively the analogues of the Josephson and CDW interactions. Their scaling dimensions with respect to the Luttingerliquid fixed point (11) are respectively $\Delta_{\perp}=1 /(2 K), \Delta_{d}=$ $2 K$. The hopping term is thus a relevant perturbation when $K>1 / 4$, and the density-density term, when $K<1$. For contact interactions, $K>1$, so the density-density term is irrelevant and a BKT transition, driven by the hopping term, leads to a superfluid phase with phase coherence between the tubes ${ }^{34}$ For $1 / 4<K<1$, which is the regime we are focusing on here, the two perturbations are strongly relevant competing perturbations. A different quantum phase transition emerges from this competition. In this case, the infrared (IR) properties of the system is governed by the effective Hamiltonian:

$$
\begin{aligned}
\mathcal{H}_{\mathrm{eff}} & =\sum_{n=1}^{N} \int d x \frac{v}{2}\left[K\left(\partial_{x} \varphi_{n}\right)^{2}+\frac{1}{K}\left(\partial_{x} \vartheta_{n}\right)^{2}\right] \\
& -\int d x \sum_{n=1}^{N-1}\left[\frac{g_{\perp}}{a^{2}} \cos \left(\sqrt{\pi}\left(\vartheta_{n+1}-\vartheta_{n}\right)\right)\right. \\
& \left.+\frac{g_{d}}{a^{2}} \cos \left(\sqrt{4 \pi}\left(\varphi_{n+1}-\varphi_{n}\right)\right)\right],
\end{aligned}
$$

where the marginal forward scattering term has been discarded.

Our strategy thus consists in, first investigating the physical properties of model (15), and then, analysing the main effect of less relevant perturbations omitted in (15), such as the forward scattering and higher harmonics of the bosonized representations 89 .

One can anticipate on symmetry grounds that the strongly relevant terms of model (15) will not introduce a spectral gap for all degrees of freedom. Indeed, it is straightforward to see that the model is invariant under two independent global U(1) transformations:

$$
\begin{aligned}
& \varphi_{n} \rightarrow \varphi_{n}+\alpha \\
& \vartheta_{n} \rightarrow \vartheta_{n}+\beta,
\end{aligned}
$$

$\alpha, \beta$ being real numbers. Introducing left and right components of the bosonic fields $\varphi_{n L, R}=\left(\varphi_{n} \pm \vartheta_{n}\right) / 2$, the transformation (16) gives a $\mathrm{U}(1)_{L} \times \mathrm{U}(1)_{R}$ global continuous symmetry of model (15). We thus expect the model to be gapless with one bosonic field protected by the symmetry (16).

One way to reveal the emergence of this bosonic mode is to single it out by performing a change of basis on the bosons fields. In this respect, we thus introduce the bosonic field $\Phi_{0 R, L}$, and $N-1$ other fields $\Phi_{l R, L}(l=1, \ldots, N-1)$ as follows:

$$
\begin{aligned}
\Phi_{0 R(L)} & =\frac{1}{\sqrt{N}}\left(\varphi_{1}+\ldots+\varphi_{N}\right)_{R(L)} \\
\Phi_{l R(L)} & =\frac{1}{\sqrt{l(l+1)}}\left(\varphi_{1}+\ldots+\varphi_{l}-l \varphi_{l+1}\right)_{R(L)}
\end{aligned}
$$

Note that in the context of condensed matter physics, the boson $\Phi_{0 R, L}$ would be called the charge boson since it is simply the sum of all bosons and describes fluctuations of the total charge. The inverse transformation can be easily found; we do not however need its explicit form but only some of its general properties:

$$
\varphi_{n R(L)}=\frac{1}{\sqrt{N}} \Phi_{0 R(L)}+\mathbf{v}^{(n)} \cdot \boldsymbol{\Phi}_{R(L)},
$$

where $\Phi$ is the $N-1$ dimensional vector with components $\Phi_{l}$ $(l=1, \ldots, N-1)$ and the $N$ vectors $\mathbf{v}^{(n)}(n=1, \ldots, N)$ 
satisfy the following relations:

$$
\begin{aligned}
\mathbf{v}^{(n)} \cdot \mathbf{v}^{(m)} & =\delta_{n m}-\frac{1}{N} \\
\sum_{n=1}^{N} \mathbf{v}^{(n)} & =\mathbf{0} \\
\sum_{n=1}^{N} v_{p}^{(n)} v_{q}^{(n)} & =\delta_{p q},
\end{aligned}
$$

with $p, q=1, \ldots, N-1$. In the new basis, the effective Hamiltonian (15) simplifies as follows:

$$
\begin{aligned}
\mathcal{H}_{\mathrm{eff}} & =\int d x \frac{v}{2}\left[K\left(\partial_{x} \Phi_{0}\right)^{2}+\frac{1}{K}\left(\partial_{x} \Theta_{0}\right)^{2}\right] \\
& +\int d x \frac{v}{2}\left[K\left(\partial_{x} \boldsymbol{\Phi}\right)^{2}+\frac{1}{K}\left(\partial_{x} \boldsymbol{\Theta}\right)^{2}\right] \\
& -\int d x \sum_{n=1}^{N-1}\left[\frac{g_{\perp}}{a^{2}} \cos \left(\sqrt{\pi}\left(\mathbf{v}^{(n+1)}-\mathbf{v}^{(n)}\right) \cdot \boldsymbol{\Theta}\right)\right. \\
& \left.+\frac{g_{d}}{a^{2}} \cos \left(\sqrt{4 \pi}\left(\mathbf{v}^{(n+1)}-\mathbf{v}^{(n)}\right) \cdot \boldsymbol{\Phi}\right)\right],
\end{aligned}
$$

where $\Theta_{0}$ and $\Theta$ are respectively the dual fields of $\Phi_{0}$ and $\boldsymbol{\Phi}$. It is interesting to observe that the low-energy effective theory (20) has been found in total different contexts. On the one hand, the deconfining phase transition of the 2+1dimensional $\mathrm{SU}(N)$ Georgi-Glashow model is controlled by model [20]. ${ }^{40}$ On the other hand, as shown recently, it describes the Read-Rezayi sequence of non-Abelian fractional quantum Hall states. ${ }^{41}$ We explicitly observe, from Eq. 20, that the $\Phi_{0}$ field decouples from the interaction terms and remains gapless. We now need to analyse the fate of the remaining degrees of freedom. The low-energy properties of the model depend on the value of $K$ which controls the competition between the $\boldsymbol{\Phi}$ and $\Theta$ fields of Eq. (20).

When $1 / 2<K<1$, the most relevant contribution is the dual field $\Theta$ term with scaling dimension $\Delta_{\perp}=1 /(2 K)$. This field is pinned onto the minima of its sine-Gordon model in Eq. (20) with coupling constant $g_{\perp}>0$ : $\langle\boldsymbol{\Theta}\rangle=\mathbf{0}$. In contrast, the bosonic $\boldsymbol{\Phi}$ field is a strongly fluctuating field being dual to $\Theta$. The resulting phase is a superfluid phase which is described by the following leading asymptotics of equal-time correlation functions:

$$
\begin{aligned}
\left\langle\Psi_{n}^{\dagger}(x) \Psi_{m}(0)\right\rangle & \sim x^{-1 /(2 N K)} \\
\left\langle\rho_{n}(x) \rho_{m}(0)\right\rangle & \sim \rho_{0}^{2}-\frac{K}{2 N \pi^{2} x^{2}} .
\end{aligned}
$$

In particular, the $2 \pi \rho_{0}$ oscillations of the density $\rho_{n}$ are shortranged.

When $K<1 / 2$, the most relevant contribution of Eq. 20] is now the term with the bosonic field $\boldsymbol{\Phi}$, with scaling dimension $\Delta_{d}=2 K$. The bosonic field $\boldsymbol{\Phi}$ is pinned onto the minima of its sine-Gordon model with coupling constant $g_{d}>0$ : $\langle\Phi\rangle=\mathbf{0}$. In this phase, the bosonic $\Theta$ field is a strongly fluctuating field. The resulting phase is a CDW phase, or a density-wave phase, where the intratube density are all in phase:

$$
\begin{aligned}
\left\langle\Psi_{n}^{\dagger}(x) \Psi_{m}(0)\right\rangle & \sim e^{-x / \xi} \\
\left\langle\rho_{n}(x) \rho_{m}(0)\right\rangle & \sim \rho_{0}^{2}+A \frac{\cos \left(2 \pi \rho_{0} x\right)}{x^{2 K / N}},
\end{aligned}
$$

$\xi$ being the correlation length which stems from the spectral gap opened by the relevant perturbation of Eq. 20, with coupling constant $g_{d}$, and $A$ is a non-universal constant. While the bosonic creation operator has short-ranged correlations, this phase allows the formation between the tubes of a molecular superfluid instability made of $N$ bosons. Indeed, using the bosonization approach (10), we find that the operator $M^{\dagger}=\Psi_{1}^{\dagger} \Psi_{2}^{\dagger} \ldots \Psi_{N}^{\dagger}$ is described by $M^{\dagger} \sim e^{i \sqrt{\pi} \sum_{n=1}^{N} \vartheta_{n}}=$ $e^{i \sqrt{\pi N} \Theta_{0}}$. We deduce thus the leading asymptotics of molecular superfluid correlation function:

$$
\left\langle M^{\dagger}(x) M(0)\right\rangle \sim x^{-N /(2 K)} .
$$

This instability dominates the density wave (22) only if $K>$ $N / 2$. The situation is in close parallel to the formation of molecular fermionic superfluid instability in 1D multicomponent cold fermionic atoms.

For decoupled tubes, it is well known that the superfluid correlations are dominant when $K>1 / 2$ while the CDW is the leading instability for $K<1 / 2 !^{12}$ There is a smooth crossover at $K=1 / 2$ between these two regions. But as we can see from Eqs. 21, 22, here the situation is in stark contrast to the decoupled tubes case, since $K=1 / 2$ marks the onset of a zero-temperature quantum phase transition. At $K=1 / 2$, the competition between the bosonic fields and their dual is maximal since the two strongly relevant perturbations have the same scaling dimension $\Delta_{\perp}=\Delta_{d}=1$ :

$$
\begin{aligned}
\mathcal{H}_{\mathrm{eff}} & =\sum_{n=1}^{N} \int d x \frac{v}{2}\left[\left(\partial_{x} \varphi_{n}\right)^{2}+\left(\partial_{x} \vartheta_{n}\right)^{2}\right] \\
& -\int d x \sum_{n=1}^{N-1}\left[\frac{g_{\perp}}{a^{2}} \cos \left(\sqrt{2 \pi}\left(\vartheta_{n+1}-\vartheta_{n}\right)\right)\right. \\
& \left.+\frac{g_{d}}{a^{2}} \cos \left(\sqrt{2 \pi}\left(\varphi_{n+1}-\varphi_{n}\right)\right)\right] .
\end{aligned}
$$

\section{B. Non-Abelian symmetry}

The effective Hamiltonian (24) at $K=1 / 2$ displays a hidden SU(2) symmetry when $g_{\perp}=g_{d} \cdot \frac{38139}{1}$ In this respect, it is worth introducing the following family of SU(2) matrices:

$$
g_{n}=\frac{1}{\sqrt{2}}\left(\begin{array}{cc}
e^{-i \sqrt{2 \pi} \varphi_{n}} & i e^{-i \sqrt{2 \pi} \vartheta_{n}} \\
i e^{i \sqrt{2 \pi} \vartheta_{n}} & e^{i \sqrt{2 \pi} \varphi_{n}}
\end{array}\right),
$$

with $n=1, \ldots, N$. The interaction part, $V_{\text {eff }}$, of model (24) can be expressed in terms of these matrices:

$$
\begin{aligned}
V_{\mathrm{eff}} & =\int d x \frac{1}{2 a^{2}}\left[-\left(g_{\perp}+g_{d}\right) \operatorname{Tr}\left(g_{n+1}^{\dagger} g_{n}\right)\right. \\
& \left.+\left(g_{\perp}-g_{d}\right) \operatorname{Tr}\left(g_{n+1}^{\dagger} \sigma_{z} g_{n} \sigma_{z}\right)\right],
\end{aligned}
$$


$\sigma_{z}$ being the third Pauli matrix. We conclude that the interaction term (26) displays a non-Abelian symmetry $\mathrm{SU}(2)_{L} \times$ $\mathrm{SU}(2)_{R}$ for $g_{\perp}=g_{d}$ since it is then invariant under the symmetry: $g_{n} \rightarrow U g_{n} V, U$ and $V$ being two independent SU(2) matrices. When $g_{\perp} \neq g_{d}$, due to the last term of Eq. (26), this non-Abelian symmetry is broken down to $\mathrm{U}(1)_{L} \times \mathrm{U}(1)_{R}$ in full agreement with Eq. (16).

It is then natural to expect that the quantum phase transition between the superfluid and CDW phase occurs precisely at $g_{\perp}=g_{d}$ where the effective Hamiltonian 24 enjoys an enlarged $\mathrm{SU}(2)_{L} \times \mathrm{SU}(2)_{R}$ global symmetry as well as a selfdual symmetry $\varphi_{n} \leftrightarrow \vartheta_{n}$ :

$$
\begin{aligned}
\mathcal{H}^{*} & =\sum_{n=1}^{N} \int d x \frac{v}{2}\left[\left(\partial_{x} \varphi_{n}\right)^{2}+\left(\partial_{x} \vartheta_{n}\right)^{2}\right] \\
& -\frac{g_{d}}{a^{2}} \int d x \sum_{n=1}^{N-1}\left[\cos \left(\sqrt{2 \pi}\left(\vartheta_{n+1}-\vartheta_{n}\right)\right)\right. \\
& \left.+\cos \left(\sqrt{2 \pi}\left(\varphi_{n+1}-\varphi_{n}\right)\right)\right]
\end{aligned}
$$

The existence of this global $\mathrm{SU}(2)_{L} \times \mathrm{SU}(2)_{R}$ continuous symmetry leads us to expect that model 27) displays quantum critical properties which are governed by some CFT. Due to the $\mathrm{SU}(2)_{L} \times \mathrm{SU}(2)_{R}$ invariance, the natural candidate is the $\mathrm{SU}(2)_{k}$ CFT with central charge $c=3 k /(k+2)$, where $k$ is some integer to be determined. In the next subsection, we are going to show non-perturbatively that the model (27) is indeed conformally invariant for all sign of $g_{d}$, and belongs to the $\mathrm{SU}(2)_{N}$ universality class.

\section{Conformal embedding approach}

Let us now consider the CFTs underlying the physics of our system at the Luttinger-liquid fixed point, i.e. when the intertube interactions are turned off, $g_{\perp}=g_{d}=0$.

At $K=1 / 2$, each boson field $\varphi_{n}(n=1, \ldots, N)$ is a compactified boson with radius $R=1 / \sqrt{2 \pi}: \varphi_{n} \sim \varphi_{n}+2 \pi R$, $\vartheta_{n} \sim \vartheta_{n}+2 \pi R$. This equivalence can be viewed as gauge redundancy in the description since the density and creation operators 89 ) for $K=1 / 2$ are invariant under that transformation. At this special radius, each boson field $\varphi_{n}$ describes an SU(2) $)_{1}$ CFT with central charge $c=1$. These CFTs are each generated by left and right $\mathrm{SU}(2)_{1}$ currents $\mathbf{j}_{n R, L}$ which read as follows in terms of the left-right moving bosons (see Appendix Sec. 1 for more details):

$$
\begin{aligned}
j_{n R, L}^{\dagger} & =\frac{1}{2 \pi a} \exp \left(\mp i \sqrt{8 \pi} \varphi_{n R, L}\right) \\
j_{n R, L}^{z} & =\frac{1}{\sqrt{2 \pi}} \partial_{x} \varphi_{n R, L} .
\end{aligned}
$$

The SU(2) matrix (25) is the spin-1/2 SU(2) 1 primary operator with scaling dimension $1 / 2$. The conformal symmetry of the non-interacting part of model (27) is then $\mathrm{SU}(2)_{1} \times \mathrm{SU}(2)_{1} \times$ $\ldots \times \mathrm{SU}(2)_{1}$ with central charge $N$ (i.e., $N$ gapless bosonic modes). The $N$ SU(2) 1 currents (28) can then be combined to form an $\mathrm{SU}(2)_{N}$ current:

$$
\mathbf{I}_{R, L}=\sum_{n=1}^{N} \mathbf{j}_{n R, L}
$$

The next step of the approach is to go from these $N \mathrm{SU}(2)_{1}$ CFTs to the $\mathrm{SU}(2)_{N}$ CFT with central charge $3 N /(N+2)$. In this respect, one can use the following conformal embedding to investigate the critical properties of model (27):

$$
\mathrm{SU}(2)_{1} \times \mathrm{SU}(2)_{1} \times \cdots \times \mathrm{SU}(2)_{1} \rightarrow \mathrm{SU}(2)_{N} \times \mathcal{G}_{N},
$$

where $\mathcal{G}_{N}$ is a discrete CFT with central charge $c_{\mathcal{G}_{N}}=N-$ $3 N /(N+2)=N(N-1) /(N+2)$. It is important to note that the latter central charge coincides with the sum of the central charges of the $N-1$ first minimal models. 40

$$
c_{\mathcal{G}_{N}}=\frac{N(N-1)}{(N+2)}=\sum_{m=2}^{N+1}\left(1-\frac{6}{m(m+1)}\right) .
$$

The $\mathcal{G}_{N}$ CFT is thus related to the product $\mathcal{M}_{3} \times \mathcal{M}_{4} \times \ldots \times$ $\mathcal{M}_{N+1}$ where $\mathcal{M}_{p}$ denotes the minimal model series with central charge $c_{p}=1-6 / p(p+1)$; for $p=3,4,5$ they respectively correspond to the Ising, TIM (Tricritical Ising Model), and $\mathbb{Z}_{3}$ Potts CFTs ${ }^{15}$ The precise identification requires a projection P: $\mathcal{G}_{N} \sim P\left(\mathcal{M}_{3} \times \mathcal{M}_{4} \ldots \times \mathcal{M}_{N+1}\right)$, which has been described in $\operatorname{Ref} 48$.

The central point of our analysis is to show that the self-dual perturbation of model 27):

$\mathcal{V}_{\mathrm{sd}}=\sum_{n=1}^{N-1} \cos \left(\sqrt{2 \pi}\left(\varphi_{n+1}-\varphi_{n}\right)\right)+\cos \left(\sqrt{2 \pi}\left(\vartheta_{n+1}-\vartheta_{n}\right)\right)$

does not depend on the $\mathrm{SU}(2)_{N}$ CFT. More precisely, we show, in Sec. 2 of the Appendix, that the operator $\mathcal{V}_{\mathrm{sd}}$ is indeed a singlet under the $\mathrm{SU}(2)_{N}$ CFT and a primary operator of the $\mathcal{G}_{N}$ CFT with a scaling dimension equal to one. Hence, since it is a strongly relevant perturbation, $\mathcal{V}_{\text {sd }}$ introduces a spectral gap for the discrete $\mathcal{G}_{N}$ degrees of freedom, but leaves the $\mathrm{SU}(2)_{N}$ ones intact. We thus conclude that model (27) displays critical properties in the $\mathrm{SU}(2)_{N}$ universality class for all signs of $g_{d}$.

However, we are not guaranteed that the critical properties of the initial model (1) are controlled by this $\mathrm{SU}(2)_{N}$ fixed point. One has to investigate the stability of the latter fixed point with respect to the marginal forward scattering operator of Eq. (12) and the contributions of the decoupled tubes limit such as the higher harmonics of the bosonized description (89) that we have neglected. The marginal forward-scattering process breaks the non-abelian $\mathrm{SU}(2)_{L} \times \mathrm{SU}(2)_{R}$ symmetry for $g_{\perp}=g_{d}$ down to $\mathrm{U}(1)_{L} \times \mathrm{U}(1)_{R}$. In this respect, the symmetry of the IR fixed point is then $\mathrm{U}(1) \times \mathbb{Z}_{N}$ since $\mathrm{SU}(2)_{N} / \mathrm{U}(1) \sim \mathbb{Z}_{N}{ }^{[2324}$ The latter CFT with central charge $c_{N}=2(N-1) /(N+2)$ is called the $\mathbb{Z}_{N}$ parafermionic CFT and describes the multicritical properties of $2 \mathrm{D} \mathbb{Z}_{N}$ generalization of Ising models. ${ }^{[23}$ The self-duality symmetry, $\varphi_{n} \leftrightarrow \vartheta_{n}$, of model 27] corresponds to the Kramers-Wannier (KW) self-duality symmetry of $\mathbb{Z}_{N}$ models. In fact, the $\mathbb{Z}_{N}$ 
symmetry can be defined directly in terms of the bosons at $K=1 / 2$ :

$$
\begin{aligned}
\vartheta_{n} & \rightarrow \vartheta_{n}+\frac{\sqrt{2 \pi}}{N} m \\
\varphi_{n} & \rightarrow \varphi_{n},
\end{aligned}
$$

with $m=0,1, \ldots, N-1$. Using the representation (9), this $\mathbb{Z}_{N}$ symmetry has a simple interpretation in terms of the original bosonic creation operators:

$$
\Psi_{n}^{\dagger}(x) \rightarrow \Psi_{n}^{\dagger}(x) e^{i \frac{2 \pi}{N} m} .
$$

In general, the $\mathrm{SU}(2)_{N}$ and $\mathbb{Z}_{N}$ fixed points are fragile since they can be destabilized by several relevant primary operators. Here, the presence of the $\mathbb{Z}_{N}$ symmetry (33) does protect the system from several relevant perturbations. However, primary operators such as the thermal operators $\epsilon_{k}$, with scaling dimensions $\Delta_{k}=2 k(k+1) /(N+2)$, with $k=1, \ldots[N / 2]$ $([N / 2]$ is the integer part of $N / 2)$, are invariant under the $\mathbb{Z}_{N}$ symmetry and might destabilize the IR fixed point. Fortunately, some of these operators, such as $\epsilon_{1}$, can be killed by a special fine-tuning of the coupling constants by imposing the self-duality symmetry. Indeed, under the KW symmetry, one has $\epsilon_{k} \rightarrow-\epsilon_{k}$, and $\epsilon_{1}$ disappears along the self-dual manifold the model. As a result, for small values of $N$, the $\mathrm{SU}(2)_{N}$ or $\mathrm{U}(1) \times \mathbb{Z}_{N}$ fixed point is likely to be stable thanks to the control of the dipole strength and the intertube hopping. In contrast, for $N \geq 5$, a strongly relevant self-dual perturbation $\epsilon_{2}$ with scaling dimension $12 /(N+2)$ will be generated in the $\mathbb{Z}_{N}$ sector. The resulting field theory which captures the quantum phase transition in this case becomes:

$$
\mathcal{S}_{\text {eff }}=\mathcal{S}_{\mathbb{Z}_{N}}+\lambda \int d^{2} x \epsilon_{2}(x),
$$

where $\mathcal{S}_{\mathbb{Z}_{N}}$ stands for the action of the $\mathbb{Z}_{N}$ CFT. Model (35) turns out to be an integrable deformation of the $\mathbb{Z}_{N}$ CFT $49 \overline{50}$ The nature of the phase transition depends on the sign of the coupling constant $\lambda{ }^{49[50}$ For $\lambda<0$, the field theories 35 are massive and the phase transition is of first-order type. For $\lambda>$ 0 it is known that model (35) has a massless renormalizationgroup flow onto a BKT U(1) gapless phase with central charge $c=1$. In the latter case, there is an intermediate gapless phase between the superfluid and CDW phases for $N \geq 5$. Adding the contribution of the gapless mode $\Phi_{0}$, this phase displays extended quantum criticality with central charge $c=2$. Unfortunately, our approach does not enable us to fix the sign of the perturbation of model (35) and we cannot discriminate between the two possibilities for the nature of the transition for $N \geq 5$. In the following, we will investigate the nature of the quantum phase transition for small values of $N$, i.e., $N=2,3$, which respectively correspond to double-tube and triple-tube systems.

\section{DOUBLE-TUBE SYSTEM}

In this section, we determine the zero-temperature physical properties of the double-tube system which corresponds to model (1) with $N=2$ (see Fig. (2)).
The harmonic-fluid representation (11, 12, simplifies as follows in the $N=2$ case:

$$
\begin{aligned}
\mathcal{H}_{N=2} & =\int d x \frac{v}{2}\left[K\left(\partial_{x} \varphi_{1}\right)^{2}+\frac{1}{K}\left(\partial_{x} \vartheta_{1}\right)^{2}+1 \rightarrow 2\right] \\
& -\int d x\left[\frac{g_{\perp}}{a^{2}} \cos \left(\sqrt{\pi}\left(\vartheta_{2}-\vartheta_{1}\right)\right)\right. \\
& \left.+\frac{g_{d}}{a^{2}} \cos \left(\sqrt{4 \pi}\left(\varphi_{2}-\varphi_{1}\right)\right)\right] \\
& +\lambda \int d x \partial_{x} \varphi_{2} \partial_{x} \varphi_{1} .
\end{aligned}
$$

The next step is to use the change of basis (17):

$$
\begin{aligned}
\Phi_{0 R(L)} & =\frac{1}{\sqrt{2}}\left(\varphi_{1}+\varphi_{2}\right)_{R(L)} \\
\Phi_{1 R(L)} & =\frac{1}{\sqrt{2}}\left(\varphi_{1}-\varphi_{2}\right)_{R(L)},
\end{aligned}
$$

so that we obtain

$$
\begin{aligned}
& \mathcal{H}_{N=2}=\int d x \frac{v_{0}}{2}\left[K_{0}\left(\partial_{x} \Phi_{0}\right)^{2}+\frac{1}{K_{0}}\left(\partial_{x} \Theta_{0}\right)^{2}\right] \\
+ & \int d x \frac{v_{1}}{2}\left[K_{1}\left(\partial_{x} \Phi_{1}\right)^{2}+\frac{1}{K_{1}}\left(\partial_{x} \Theta_{1}\right)^{2}\right] \\
- & \int d x\left[\frac{g_{\perp}}{a^{2}} \cos \left(\sqrt{2 \pi} \Theta_{1}\right)+\frac{g_{d}}{a^{2}} \cos \left(\sqrt{8 \pi} \Phi_{1}\right)\right]
\end{aligned}
$$

There is a velocity anisotropy and two different Luttinger parameters which stem from the marginal term of Eq. (36) with coupling constant $\lambda$ :

$$
\begin{aligned}
& K_{0}=\frac{K}{\sqrt{1+\lambda v / K}} \\
& K_{1}=\frac{K}{\sqrt{1-\lambda v / K}} .
\end{aligned}
$$

The effective Hamiltonian (38) has been first studied in Ref 51 in the context of a bosonic two-leg ladder for incommensurate filling. It separates into two commuting pieces: $\mathcal{H}_{N=2}=$ $\mathcal{H}_{0}+\mathcal{H}_{1}\left(\left[\mathcal{H}_{0}, \mathcal{H}_{1}\right]=0\right)$. The first contribution $\mathcal{H}_{0}$ of Eq. (38) has the form of a Tomonaga-Luttinger Hamiltonian; this signals that the bosonic field $\Phi_{0}$ is a gapless degree of freedom. All the non-trivial physics is encoded in $\mathcal{H}_{1}$ which describes the competition between the intertube hopping and dipole-dipole interactions. As already discussed in Sec. II in the general $N$ case, there are two different phases which stem from this competition. When $1 / 2<K_{1}<1, \mathcal{H}_{1}$ has a spectral gap $\Delta_{1}$ due to the cosine term with the bosonic field $\Theta_{1}$. As a result, $\Theta_{1}$ is pinned onto one of the minima of its sine-Gordon model, which we can choose to be $\left\langle\Theta_{1}\right\rangle=0$ $\left(g_{\perp}>0\right)$. Thus, at low-energy $E \ll \Delta_{1}$, the creation operator of the bosons simplifies as follows: $\Psi_{n}^{\dagger} \sim \sqrt{\rho_{0}} e^{i \sqrt{\pi / 2} \Theta_{0}}$, while the $2 \pi \rho_{0}$ density operator is short-ranged since it depends on the strongly fluctuating field $\Phi_{1}$. The resulting superfluid phase is then characterized by:

$$
\begin{aligned}
\left\langle\Psi_{n}^{\dagger}(x) \Psi_{m}(0)\right\rangle & \sim x^{-1 /\left(4 K_{0}\right)} \\
\left\langle\rho_{n}(x) \rho_{m}(0)\right\rangle & \sim \rho_{0}^{2}-\frac{K_{0}}{4 \pi^{2} x^{2}}
\end{aligned}
$$




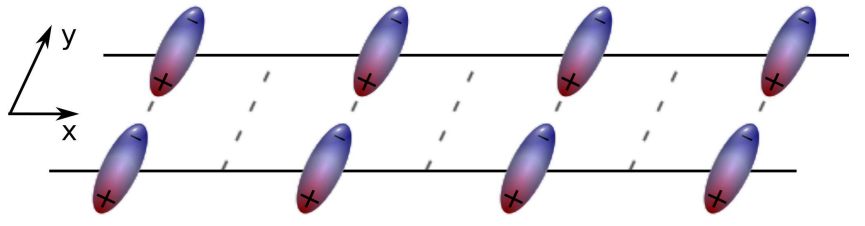

FIG. 2: (color online) The CDW order characterized by Eq. (43); the two CDW orders in each tube are in phase.

with $n, m=1,2$. In fact, in this phase, one may also consider the symmetric combination of the density with $4 \pi \rho_{0}$ oscillations in each tube:

$$
\begin{aligned}
\rho^{4 \pi \rho_{0}} & \sim \rho_{0} \sum_{n=1}^{2} \cos \left(4 \pi \rho_{0} x+4 \sqrt{\pi} \varphi_{n}\right) \\
& \sim 2 \rho_{0} \cos \left(4 \pi \rho_{0} x+\sqrt{8 \pi} \Phi_{0}\right) \cos \left(\sqrt{8 \pi} \Phi_{1}\right) .
\end{aligned}
$$

This operator is naively short-ranged in the phase with $\left\langle\Theta_{1}\right\rangle=$ 0 since it depends on the field $\Phi_{1}$. However, first-order perturbation theory with the Hamiltonian $\mathcal{H}_{1}$ cancels the contribution of $\cos \left(\sqrt{8 \pi} \Phi_{1}\right)$ in $\rho^{4 \pi \rho_{0}}$. The low-energy description of that operator in the superfluid phase thus simplifies as follows:

$$
\rho^{4 \pi \rho_{0}} \sim 2 \rho_{0} \cos \left(4 \pi \rho_{0} x+\sqrt{8 \pi} \Phi_{0}\right) .
$$

We deduce that the two-point correlation function of that operator has a power-law decay with exponent $4 K_{0}$. Since $1 / 2<K_{0}<1$, the decay is much slower than the decays of the instabilities of Eq. (40).

The second phase is obtained when $K_{1}<1 / 2$. The most relevant contribution of Eq. (38) is now the cosine term with the bosonic field $\Phi_{1}$ which is pinned: $\left\langle\Phi_{1}\right\rangle=0\left(g_{d}>0\right)$. In this phase, the dual field $\Theta_{1}$ is a strongly fluctuating field. The resulting phase is a CDW phase where the two $2 \pi \rho_{0} \mathrm{CDW}$ on each tube are in phase (see Fig. 2):

$$
\begin{aligned}
\left\langle\Psi_{n}^{\dagger}(x) \Psi_{m}(0)\right\rangle & \sim e^{-x / \xi} \\
\left\langle\rho_{n}(x) \rho_{m}(0)\right\rangle & \sim \rho_{0}^{2}+A \frac{\cos \left(2 \pi \rho_{0} x\right)}{x^{K_{0}}},
\end{aligned}
$$

$A$ being a non-universal constant and $\xi \sim v_{1} / \Delta_{1}$ is the correlation length which stems from the spectral gap of the Hamiltonian $\mathcal{H}_{1}$. In this phase, the dimer instability $M^{\dagger}=\Psi_{1}^{\dagger} \Psi_{2}^{\dagger} \sim$ $\rho_{0} e^{i \sqrt{2 \pi} \Theta_{0}}$ has a power-law correlation function with a larger decay $1 / K_{0}\left(K_{0}<1\right)$.

The quantum phase transition between these two different phases occurs at $K_{1}=1 / 2$. From Eq. (39), we observe that since $\lambda<0$, we have $K_{1}<K$, i.e., attractive intertube interactions such as in Eq. (7) are favorable in order to reach the transition. The effective Hamiltonian which governs this transition writes:

$$
\begin{gathered}
\mathcal{H}_{\mathrm{eff}}=\int d x \frac{v_{1}}{2}\left[\left(\partial_{x} \Phi_{1}\right)^{2}+\left(\partial_{x} \Theta_{1}\right)^{2}\right] \\
-\int d x\left[\frac{g_{\perp}}{a^{2}} \cos \left(\sqrt{4 \pi} \Theta_{1}\right)+\frac{g_{d}}{a^{2}} \cos \left(\sqrt{4 \pi} \Phi_{1}\right)\right] .
\end{gathered}
$$

This model is well known (see e.g. Ref 52) and can be exactly diagonalized by introducing two Majorana fermions $\xi^{1,2}$. This procedure is nothing but the standard bosonization of two Ising models. ${ }^{16153154}$ The refermionization rules are given by

$$
\begin{aligned}
\xi_{R}^{1}+i \xi_{R}^{2} & =\frac{1}{\sqrt{\pi a}} \exp \left(i \sqrt{4 \pi} \Phi_{1 R}\right), \\
\xi_{L}^{1}+i \xi_{L}^{2} & =\frac{1}{\sqrt{\pi a}} \exp \left(-i \sqrt{4 \pi} \Phi_{1 L}\right),
\end{aligned}
$$

where the anticommutation between the right and left-moving Majorana fermions is taken into account by the prescription: $\left[\Phi_{1 R}, \Phi_{1 L}\right]=i / 4$. The effective Hamiltonian can then be refermionized:

$$
\begin{gathered}
\mathcal{H}_{\text {eff }}=-\frac{i v_{1}}{2} \int d x \sum_{a=1}^{2}\left(\xi_{R}^{a} \partial_{x} \xi_{R}^{a}-\xi_{L}^{a} \partial_{x} \xi_{L}^{a}\right) \\
-\int d x\left[i m_{1} \xi_{R}^{1} \xi_{L}^{1}+i m_{2} \xi_{R}^{2} \xi_{L}^{2}\right],
\end{gathered}
$$

where the two masses are given by:

$$
\begin{aligned}
& m_{1}=\left(g_{d}-g_{\perp}\right) / a \\
& m_{2}=\left(g_{d}+g_{\perp}\right) / a .
\end{aligned}
$$

The effective model (44) at $K_{1}=1 / 2$ is thus mapped onto two decoupled 1D Ising models in a transverse field. The Majorana fermion $\xi_{R, L}^{2}$ always has a positive mass $m_{2}$ which means that, in our convention, the corresponding Ising model belongs to its disordered phase where the disorder operator condenses: $\left\langle\mu_{2}\right\rangle \neq 0$. In contrast, the Majorana fermion $\xi_{R, L}^{1}$ has a smaller mass $m_{1}$ which can change its sign; by tuning the hopping $g_{\perp}$, we are thus able to reach the Ising $\mathbb{Z}_{2}$ quantum critical point where $m_{1}=0$.

The different order parameters defined in Eqs. 40, 43, can be expressed in terms of the Ising order $\left(\sigma_{1,2}\right)$ and disorder $\left(\mu_{1,2}\right)$ parameters of the two underlying Ising models. In this respect, we need the Ising description of all the vertex operators with a scaling dimension equal to $1 / 4$

$$
\begin{aligned}
& \sqrt{2} \cos \left(\sqrt{\pi} \Phi_{1}\right) \sim \mu_{1} \mu_{2}, \sqrt{2} \cos \left(\sqrt{\pi} \Theta_{1}\right) \sim \sigma_{1} \mu_{2} \\
& \sqrt{2} \sin \left(\sqrt{\pi} \Phi_{1}\right) \sim \sigma_{1} \sigma_{2}, \sqrt{2} \sin \left(\sqrt{\pi} \Theta_{1}\right) \sim \mu_{1} \sigma_{2},
\end{aligned}
$$

where the Ising order parameters are normalized at the $\mathbb{Z}_{2}$ quantum critical point according to:

$$
\left\langle\sigma_{a}(\tau, x) \sigma_{b}(0,0)\right\rangle=\frac{\delta_{a b}}{\left(v_{1}^{2} \tau^{2}+x^{2}\right)^{1 / 8}},
$$

with $a, b=1,2$, and similarly for the two-point correlations of the Ising disorder operators. At low-energy, i.e., $E \ll m_{2}$, we can average the operators of the second Ising model $\left(\left\langle\mu_{2}\right\rangle \neq 0\right.$ and $\left.\left\langle\sigma_{2}\right\rangle=0\right)$. The bosonic creation and density operators then simplify as follows, using Eq. [48):

$$
\begin{aligned}
& \Psi_{n}^{\dagger} \sim e^{i \sqrt{\pi / 2 K_{0}} \Theta_{0}} \sigma_{1} \\
& \rho_{n} \sim \rho_{0}+\sqrt{\frac{K_{0}}{2 \pi}} \partial_{x} \Phi_{0} \\
& \quad+\sqrt{A} \cos \left[2 \pi \rho_{0} x+\sqrt{2 \pi K_{0}} \Phi_{0}\right] \mu_{1}
\end{aligned}
$$


with $K_{0} \simeq 1 / 2$. In particular, we observe that the bosonic creation operator expresses in terms of the Ising order operator $\sigma_{1}$, in full agreement with the identification of the discrete $\mathbb{Z}_{N}$ symmetry (34) made in Sec. II.

It is then straightforward to deduce the zero-temperature phase diagram of model (44). When $g_{\perp}<g_{d}$, i.e. $m_{1}>0$, the Ising model belongs to its disorder phase with $\left\langle\sigma_{1}\right\rangle=0$ and $\left\langle\mu_{1}\right\rangle \neq 0$. We recover the CDW phase with the leading asymptotics (43). When $g_{\perp}>g_{d}$, the Ising model belongs to its ordered phase with $\left\langle\sigma_{1}\right\rangle \neq 0$ and the double-tube system sits in its superfluid phase (40). The quantum phase transition between these two phases occurs for $g_{\perp}=g_{d}$ and is driven by the Ising degrees of freedom. The resulting critical properties are determined by the low-energy description 50 and correlations 49]:

$$
\begin{aligned}
\left\langle\Psi_{n}^{\dagger}(x) \Psi_{m}(0)\right\rangle & \sim x^{-1 /\left(4 K_{0}\right)-1 / 4} \\
\left\langle\rho_{n}(x) \rho_{m}(0)\right\rangle & \sim \rho_{0}^{2}-\frac{K_{0}}{4 \pi^{2} x^{2}}+A \frac{\cos \left(2 \pi \rho_{0} x\right)}{x^{K_{0}+1 / 4}}
\end{aligned}
$$

with $K_{0} \simeq 1 / 2$. The quantum phase transition is thus described by a $\mathrm{U}(1) \times \mathbb{Z}_{2}$ CFT with a central charge $c=$ $1+1 / 2=3 / 2$. The $\mathrm{SU}(2)_{2}$ critical properties of the perturbation (32) for $N=2$ are weakly broken down to $\mathrm{U}(1) \times$ $\mathbb{Z}_{2}$ since the bosonic fields $\Phi_{0}$ and $\Phi_{1}$ have different Luttinger parameters (39) and velocities.

\section{TRIPLE-TUBE SYSTEMS}

In this section, we determine the zero-temperature physical properties of the triple-tube system which corresponds to model (1) with $N=3$ (see Fig. 3). We consider here two different geometries: one with open-boundary conditions (Fig. 3 a)) and the other with periodic boundary conditions, where the tubes form an equilateral triangle (see Fig. 3. b)). In the latter case, we assume a non-frustrated situation where all intertube density-density interactions are attractive.

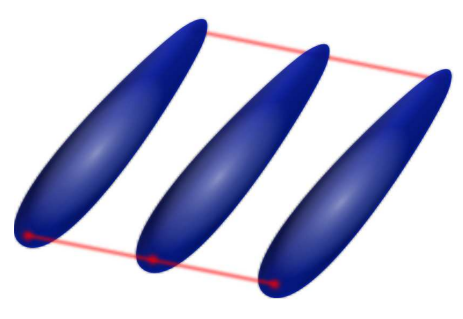

(a)

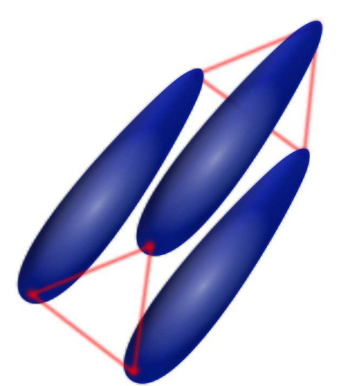

(b)
FIG. 3: A triple-tube system for (a) open boundary conditions; (b) periodic boundary conditions. As in Fig. 1. the tubes are separated by a distance $d$.

\section{A. $\mathbb{Z}_{3}$ emerging quantum criticality}

The harmonic-fluid representation for the triple-tube system of Fig. 3.a) is given by model $(11,12$ with $N=3$. Here, we follow the general strategy of Sec. II and first consider the most relevant contribution, i.e. Eq. 15 with $N=3$. We single out the gapless U(1) $\Phi_{0}$ bosonic field by switching to the basis (17) with $N=3$. The inverse transformation (18) explicitly reads as follows:

$$
\begin{aligned}
\varphi_{1} & =\frac{1}{\sqrt{3}} \Phi_{0}+\frac{1}{\sqrt{2}} \Phi_{1}+\frac{1}{\sqrt{6}} \Phi_{2} \\
\varphi_{2} & =\frac{1}{\sqrt{3}} \Phi_{0}-\frac{2}{\sqrt{6}} \Phi_{2} \\
\varphi_{3} & =\frac{1}{\sqrt{3}} \Phi_{0}-\frac{1}{\sqrt{2}} \Phi_{1}+\frac{1}{\sqrt{6}} \Phi_{2},
\end{aligned}
$$

with similar definitions for the dual fields $\vartheta_{1,2,3}$. The effective Hamiltonian 15 for $N=3$ separates into two commuting pieces: $\mathcal{H}_{\text {eff }}=\mathcal{H}_{0}+\mathcal{H}_{s}$, where $\mathcal{H}_{0}$ takes the form of the Tomonaga-Luttinger model:

$$
\mathcal{H}_{0}=\int d x \frac{v}{2}\left[K\left(\partial_{x} \Phi_{0}\right)^{2}+\frac{1}{K}\left(\partial_{x} \Theta_{0}\right)^{2}\right] .
$$

The remaining degrees of freedom in $\mathcal{H}_{s}$ are strongly coupled and control the quantum phase transition between the superfluid phase 21) and the CDW phase 22, at $K=1 / 2$. When $K=1 / 2$ and in the new basis, the effective Hamiltonian for these degrees of freedom is given by:

$$
\begin{aligned}
\mathcal{H}_{s}=\int d x \frac{v}{2}\left[\left(\partial_{x} \Phi_{1}\right)^{2}+\left(\partial_{x} \Theta_{1}\right)^{2}+1 \rightarrow 2\right] \\
-\int d x\left[\frac{2 g_{\perp}}{a^{2}} \cos \left(\sqrt{\pi} \Theta_{1}\right) \cos \left(\sqrt{3 \pi} \Theta_{2}\right)\right. \\
\left.+\frac{2 g_{d}}{a^{2}} \cos \left(\sqrt{\pi} \Phi_{1}\right) \cos \left(\sqrt{3 \pi} \Phi_{2}\right)\right] .
\end{aligned}
$$

The bosonic field $\Phi_{1}$ is at the free-fermion point, i.e. the terms of the form $\cos \left(\beta \Phi_{1}\right)$ or $\cos \left(\beta \Theta_{1}\right)$ that appear in the Hamiltonian all have $\beta=\sqrt{\pi}$. For this reason, we can introduce two effective Ising models with order parameters $\sigma_{1,2}$ and disorder fields $\mu_{1,2}$, so as to refermionize the vertex operators of Eq. (54) with a scaling dimension $1 / 4$, just as we did for the double-tube system (see Eq. (48)):

$$
\begin{aligned}
& \mathcal{H}_{s}=-\frac{i v}{2} \int d x \sum_{a=1}^{2}\left(\xi_{R}^{a} \partial_{x} \xi_{R}^{a}-\xi_{L}^{a} \partial_{x} \xi_{L}^{a}\right) \\
& +\int d x \frac{v}{2}\left[\left(\partial_{x} \Phi_{2}\right)^{2}+\left(\partial_{x} \Theta_{2}\right)^{2}\right] \\
& -\frac{\sqrt{2}}{a} \int d x\left[g_{\perp} \sigma_{1} \mu_{2}: \cos \left(\sqrt{3 \pi} \Theta_{2}\right):\right. \\
& \left.+g_{d} \mu_{1} \mu_{2}: \cos \left(\sqrt{3 \pi} \Phi_{2}\right):\right]
\end{aligned}
$$

The next step of the approach is to switch to a different basis to determine the main effect of the relevant perturbation of 
model (55). In this respect, we exploit the fact that the CFT defined by the product of one Ising CFT (the one with disorder operator $\mu_{1}$ ) and the CFT associated to the boson $\Phi_{2}$, can be described in terms of the product $\mathcal{M}_{4} \times \mathcal{M}_{5}$ CFT, i.e., TIM $\times \mathbb{Z}_{3}$ Potts CFTs. Indeed, the TIM and the $\mathbb{Z}_{3}$ Potts CFTs have central charges $c=7 / 10$ and $c=4 / 5$ respectively, so that the sum gives $c=3 / 2$, the total central charge of an Ising CFT $c=1 / 2$ and a CFT associated with one boson $c=1$. The TIM CFT describes the physical properties of the twodimensional dilute Ising model at its tricritical point ${ }^{15[58}$ The precise identification of the conformal embedding has been derived by the authors of $\operatorname{Ref} 48$ and requires a projection which restricts the primary operators of $\mathcal{M}_{4} \times \mathcal{M}_{5}$ to the subset: $\left\{\Phi_{r, s}^{(4)} \Phi_{s, q}^{(5)}\right\}$ where $\Phi_{r, s}^{(p)}(1 \leq r \leq p-1,1 \leq s \leq p)$ denotes the primary fields of the minimal model series $\mathcal{M}_{p}$ CFT with scaling dimensions $\Delta_{r, s}^{(p)}=\frac{[(p+1) r-p s]^{2}-1}{2 p(p+1)}[15]$ In Sec. 3 of the Appendix, we show that we have the following correspondences:

$$
\begin{aligned}
& \mu_{1}: \cos \left(\sqrt{3 \pi} \Phi_{2}\right):+\sigma_{1}: \cos \left(\sqrt{3 \pi} \Theta_{2}\right): \sim \sigma_{\mathrm{TIM}}^{\prime}(56) \\
& \mu_{1}: \cos \left(\sqrt{3 \pi} \Phi_{2}\right):-\sigma_{1}: \cos \left(\sqrt{3 \pi} \Theta_{2}\right): \sim \sigma_{\mathrm{TIM}} \epsilon_{\mathbb{Z}_{3}}
\end{aligned}
$$

$\sigma_{\text {TIM }}$ and $\sigma_{\text {TIM }}^{\prime}$ being respectively the magnetization and subleading magnetization operators of the TIM CFT with scaling dimensions $3 / 40$ and $7 / 8{ }^{[15}$ In Eq. $\sqrt{56}, \epsilon_{\mathbb{Z}_{3}}$ refers to the thermal operator of the $\mathbb{Z}_{3}$ Potts CFT with scaling dimension $4 / 5$.

With all these results, one can now express the low-energy effective Hamiltonian in terms of these new degrees of freedom:

$$
\begin{aligned}
& \mathcal{H}_{s}=-\frac{i v}{2} \int d x\left(\xi_{R}^{2} \partial_{x} \xi_{R}^{2}-\xi_{L}^{2} \partial_{x} \xi_{L}^{2}\right)+\mathcal{H}_{0}^{\mathrm{TIM}}+\mathcal{H}_{0}^{\mathbb{Z}_{3}} \\
- & \frac{\sqrt{2}}{2 a} \int d x\left[\left(g_{\perp}+g_{d}\right) \mu_{2} \sigma_{\mathrm{TIM}}^{\prime}\right. \\
+ & \left.\left(-g_{\perp}+g_{d}\right) \mu_{2} \sigma_{\mathrm{TIM}} \epsilon_{\mathbb{Z}_{3}}\right],
\end{aligned}
$$

where $\mathcal{H}_{0}^{\mathrm{TIM}}$ and $\mathcal{H}_{0}^{\mathbb{Z}_{3}}$ respectively denote the Hamiltonians of the TIM and $\mathbb{Z}_{3}$ CFTs. The operator $\mu_{2} \sigma_{\text {TIM }}^{\prime}$ is a strongly relevant perturbation with scaling dimension 1 which couples the Ising model associated to the Majorana fermion $\xi^{2}$ with the TIM CFT. The Ising critical point is destabilized upon switching on the disorder field $\mu_{2}$, while the $\sigma_{\text {TIM }}^{\prime}$ perturbation is known to be a massive integrable deformation of the TIM CFT for all signs of its coupling constant ${ }^{58}$ We deduce that a spectral gap $\Delta$ for the Ising and TIM degrees of freedom is opened as soon as the tubes are coupled. In the low-energy limit where $E \ll \Delta$, the low-energy effective Hamiltonian 57) then simplifies as

$$
\mathcal{H}_{s}=\mathcal{H}_{0}^{\mathbb{Z}_{3}}+A \int d x\left(-g_{\perp}+g_{d}\right) \epsilon_{\mathbb{Z}_{3}},
$$

$A$ being a non-universal constant. This model is a massive and integrable perturbation of the $\mathbb{Z}_{3}$ CFT ${ }^{[9}$ A spectral gap is thus generated, except at the fine-tuned point $g_{\perp}=g_{d}$ where the model displays a quantum critical behavior in the $\mathbb{Z}_{3}$ Potts universality class. The position of the critical point, i.e. $g_{\perp}=$ $g_{d}$, corresponds to the self-duality symmetry of model (54): $\Phi_{1,2} \leftrightarrow \Theta_{1,2}$.

Adding the contribution of the gapless boson $\Phi_{0}$, we deduce that the quantum phase transition of the triple-tube system is governed by the $\mathrm{SU}(2)_{3}$ CFT with central charge $c=$ $1+4 / 5=9 / 5$; this is in full agreement with the general analysis of Sec. II.

As in the $N=2$ case, the marginal forward-scattering operator $\mathcal{O}_{\mathrm{f} s}=\partial_{x} \varphi_{2}\left(\partial_{x} \varphi_{1}+\partial_{x} \varphi_{3}\right)$ is expected to introduce anisotropies and the symmetry of the quantum critical point is $U(1) \times \mathbb{Z}_{3}$. In particular, using the new basis 52 , we find that

$$
\mathcal{O}_{\mathrm{f} s}=\frac{2}{3}\left[\left(\partial_{x} \Phi_{0}\right)^{2}-\left(\partial_{x} \Phi_{2}\right)^{2}\right]-\frac{2}{3 \sqrt{2}} \partial_{x} \Phi_{0} \partial_{x} \Phi_{2} .
$$

As expected, the first term of this equation merely introduces velocity anisotropies and different Luttinger parameters for the bosons $\Phi_{0,1,2}$ as in the double-tube case. On the other hand, the last term gives a residual coupling between the gapless boson $\Phi_{0}$ and the remaining degrees of freedom. Such term has also be encountered in the analysis of the zero-temperature phase diagram of 1D three-component cold fermions; its main effect is not clear ${ }^{45}$ However, we think that the latter perturbation will not destroy the quantum criticality of the phase transition of the triple-tube model.

In any case, one can eliminate this term by considering a triple-tube system with transverse periodic boundary conditions as depicted in Fig. 3 (b). In that case, the forwardscattering perturbation of this problem is:

$$
\begin{aligned}
\mathcal{O}_{\mathrm{f} s}^{\Delta} & =\partial_{x} \varphi_{2}\left(\partial_{x} \varphi_{1}+\partial_{x} \varphi_{3}\right)+\partial_{x} \varphi_{1} \partial_{x} \varphi_{3} \\
& =\left(\partial_{x} \Phi_{0}\right)^{2}-\frac{1}{2}\left[\left(\partial_{x} \Phi_{1}\right)^{2}+\left(\partial_{x} \Phi_{2}\right)^{2}\right],
\end{aligned}
$$

so that this contribution is exhausted by velocities and Luttinger parameters renormalization. At the self-dual point $g_{\perp}=g_{d}$, the effective Hamiltonian which controls the transition of the model has a similar structure as in the open boundary case (see Eq. 57) :

$$
\begin{aligned}
\mathcal{H}_{s}^{\Delta} & =-\frac{i v}{2} \int d x\left(\xi_{R}^{2} \partial_{x} \xi_{R}^{2}-\xi_{L}^{2} \partial_{x} \xi_{L}^{2}\right)+\mathcal{H}_{0}^{\mathrm{TIM}}+\mathcal{H}_{0}^{\mathbb{Z}_{3}} \\
& -\frac{\sqrt{2} g_{d}}{a} \int d x \mu_{2} \sigma_{\mathrm{TIM}}^{\prime}-\frac{2 \pi g_{d}}{a} \int d x i \xi_{R}^{2} \xi_{L}^{2}
\end{aligned}
$$

We observe that here, the spectral gap for the Ising and TIM degrees of freedom is now explicit, due to the mass term $\left(m_{2}=2 \pi g_{d} / a\right)$ for the Majorana fermion $\xi^{2}$. Indeed, since this mass is positive, the corresponding Ising model belongs to its disordered phase with $\left\langle\mu_{2}\right\rangle \neq 0$. Averaging out these degrees of freedom, one is left with a TIM CFT perturbed by the subleading magnetization operator $\sigma_{\mathrm{TIM}}^{\prime}$, which gives a spectral gap $\frac{58}{6 e}$ conclude, as in the open case, that at low-energy, the $\mathbb{Z}_{3}$ degrees of freedom remain untouched and display quantum criticality with central charge $c=4 / 5$.

Our approach allows us to determine the leading asymptotics of the correlation functions at the quantum critical point with $U(1) \times \mathbb{Z}_{3}$ symmetry. The bosonic creation operators $\Psi_{n}^{\dagger}$ 
can be expressed in terms of the bosons 52 with $K=1 / 2$ :

$$
\begin{aligned}
\Psi_{1,3}^{\dagger} & \sim e^{i \sqrt{\pi / 3 K_{0}} \Theta_{0}} e^{ \pm i \sqrt{\pi} \Theta_{1}} e^{i \sqrt{\pi / 3} \Theta_{2}} \\
\Psi_{2}^{\dagger} & \sim e^{i \sqrt{\pi / 3 K_{0}} \Theta_{0}} e^{i \sqrt{4 \pi / 3} \Theta_{2}}
\end{aligned}
$$

with $K_{0} \simeq 1 / 2$. Using Eq. 48 and taking into account that the Ising model with disorder parameter $\mu_{2}$ sits in its disordered phase, we have

$$
\begin{aligned}
\Psi_{1,3}^{\dagger} & \sim e^{i \sqrt{\pi / 3 K_{0}} \Theta_{0}} \sigma_{1} e^{i \sqrt{\pi / 3} \Theta_{2}} \\
\Psi_{2}^{\dagger} & \sim e^{i \sqrt{\pi / 3 K_{0}} \Theta_{0}} e^{i \sqrt{4 \pi / 3} \Theta_{2}} .
\end{aligned}
$$

The next step is to express the operators $\sigma_{1} e^{i \sqrt{\pi / 3} \Theta_{2}}$ and $e^{i \sqrt{4 \pi / 3} \Theta_{2}}$, with respectively scaling dimensions $5 / 24$ and $1 / 3$, in terms of the TIM $\times \mathbb{Z}_{3}$ degrees of freedom. We find the following identification:

$$
\begin{aligned}
\sigma_{1} e^{i \sqrt{\pi / 3} \Theta_{2}} & \sim \sigma_{\mathrm{TIM}} \sigma_{\mathbb{Z}_{3}} \\
e^{i \sqrt{4 \pi / 3} \Theta_{2}} & \sim \epsilon_{\text {TIM }} \sigma_{\mathbb{Z}_{3}},
\end{aligned}
$$

$\sigma_{\mathbb{Z}_{3}}$ being the $\mathbb{Z}_{3}$ spin operator with scaling dimension $2 / 15$, and $\epsilon_{\text {TIM }}$, the thermal operator of the TIM CFT with scaling dimension $1 / 5$. Finally, at low-energy, i.e. for energies smaller than the spectral gap of the TIM degrees of freedom, the bosonic creation operators $\Psi_{n}^{\dagger}$ of the triple-tube system simplify as follows:

$$
\Psi_{n}^{\dagger} \sim e^{i \sqrt{\pi / 3 K_{0}} \Theta_{0}} \sigma_{\mathbb{Z}_{3}} .
$$

We observe that $\Psi_{n}^{\dagger}$ expresses directly in terms the $\mathbb{Z}_{3}$ spin operator in full agreement with the identification (34) in the general case. Similarly, we can find the low-energy representation of the $2 \pi \rho_{0} \mathrm{CDW}$ on each tube $\rho_{n}^{2 \pi \rho_{0}}$ at $K=1 / 2$ :

$$
\begin{aligned}
\rho_{n}^{2 \pi \rho_{0}} & \sim e^{i 2 \pi \rho_{0} x+\sqrt{4 \pi K} \varphi_{n}} \\
& \sim e^{i 2 \pi \rho_{0} x+i \sqrt{4 \pi K_{0} / 3} \Phi_{0}} \mu_{\mathbb{Z}_{3}},
\end{aligned}
$$

with $K_{0} \simeq 1 / 2$ and $\mu_{\mathbb{Z}_{3}}$ is the $\mathbb{Z}_{3}$ disorder spin operator which is dual to the $\mathbb{Z}_{3}$ spin operator $\sigma_{\mathbb{Z}_{3}}$. Using the results 65. 66, we deduce the leading asymptotics of the equal-time correlations at the quantum critical point of the triple-tube model:

$$
\begin{aligned}
\left\langle\Psi_{n}^{\dagger}(x) \Psi_{m}(0)\right\rangle & \sim x^{-1 /\left(6 K_{0}\right)-4 / 15} \\
\left\langle\rho_{n}(x) \rho_{m}(0)\right\rangle & \sim \rho_{0}^{2}-\frac{K_{0}}{6 \pi^{2} x^{2}}+A \frac{\cos \left(2 \pi \rho_{0} x\right)}{x^{2 K_{0} / 3+4 / 15}}
\end{aligned}
$$

with $K_{0} \simeq 1 / 2$. The quantum phase transition is thus described by a U(1) $\times \mathbb{Z}_{3}$ CFT with central charge $c=1+4 / 5=$ $9 / 5$.

\section{B. Stability of the quantum critical point and the $\mathbb{Z}_{3}$ chiral clock model}

The next step of the approach is to investigate the stability of this quantum critical point to generic perturbations allowed by the symmetries of the model. This includes all irrelevant perturbations, that we have neglected in the continuum description, which may change their status at the new $U(1) \times$ $\mathbb{Z}_{3}$ fixed point. The entire content of the $\mathbb{Z}_{3}$ Potts model is known ${ }^{15}$ and the thermal operator $\epsilon_{\mathbb{Z}_{3}}$ is the only relevant operator having zero conformal spin and preserving the global $\mathbb{Z}_{3}$ symmetry of the model. The presence of two independent coupling constants in the model, namely $J_{\perp}$ and $C_{d d}$, should be enough to kill this relevant operator and reach the $\mathbb{Z}_{3}$ critical point. A similar criticality has been found in $1 D$ XXZ Heisenberg chain in magnetic fields ${ }^{[59]}$ The other relevant operators of the $\mathbb{Z}_{3}$ Potts model carry a non-zero conformal spin: $\Phi_{(2 / 5,7 / 5)}$ and $\Phi_{(7 / 5,2 / 5)}$ are $\mathbb{Z}_{3}$ operators with scaling dimension $9 / 5$ and conformal spin $S= \pm 1$. These non-Lorentz invariant perturbations, if generated, might drive the system to the fixed point of the chiral three-state Potts universality class 60 ; the general low-energy effective field theory of triple-tube systems, which controls the vicinity of the $\mathbb{Z}_{3}$ fixed point, would then read as follows:

$$
\mathcal{H}_{\delta}^{\mathbb{Z}_{3}}=\mathcal{H}_{0}^{\mathbb{Z}_{3}}+\int d x \delta \Phi_{(2 / 5,7 / 5)}+\delta^{*} \Phi_{(7 / 5,2 / 5)}+\delta_{g} \epsilon_{\mathbb{Z}_{3}},
$$

where $\delta$ and $\delta_{g}=A\left(-g_{\perp}+g_{d}\right)$ are small coupling constants describing the departure from the $\mathbb{Z}_{3}$ quantum critical point. It is thus important to find out if the relevant non-scalar operators $\Phi_{(2 / 5,7 / 5)}, \Phi_{(7 / 5,2 / 5)}$ are permitted in the triple-tube systems investigated here.

In this respect, we observe that a non-zero conformal spin perturbation emerges in the continuum limit of the triple-tube system with open boundary conditions (Fig. 3(a)), when expanding the square root in the harmonic-fluid representation of the bosonic operator 9 ; for $K=1 / 2$, it reads:

$\mathcal{O}_{S= \pm 1}=\sum_{n=1}^{2}\left(\partial_{x} \varphi_{n+1}+\partial_{x} \varphi_{n}\right) \cos \left(\sqrt{2 \pi}\left(\vartheta_{n+1}-\vartheta_{n}\right)\right)$.

This term is a marginal perturbation with conformal spin $S= \pm 1$, and it is invariant under parity, since $x \rightarrow-x$, $\varphi_{n}(x) \rightarrow-\varphi_{n}(-x)$, which is indeed a symmetry of Eqs. (89). In the triple-tube model with periodic boundary conditions (Fig. 3(b)), the non-Lorentz invariant perturbation becomes:

$$
\mathcal{O}_{S= \pm 1}^{\Delta}=\sum_{n=1}^{3}\left(\partial_{x} \varphi_{n+1}+\partial_{x} \varphi_{n}\right) \cos \left(\sqrt{2 \pi}\left(\vartheta_{n+1}-\vartheta_{n}\right)\right)
$$

Using the basis (52) and the refermionization 45, 48, we focus on the following combinations for each triple-tube system:

$$
\begin{aligned}
& \mathcal{O}_{S= \pm 1}^{(1)}=\frac{1}{\sqrt{2}} \mu_{2} \sigma_{1}: \partial_{x} \Phi_{2} \cos \left(\sqrt{3 \pi} \Theta_{2}\right): \\
& \mathcal{O}_{S= \pm 1}^{\Delta}=\mathcal{O}_{S= \pm 1}^{(1)}+\partial_{x} \Phi_{2} i \pi \xi_{R}^{1} \xi_{L}^{1},
\end{aligned}
$$

where the second operator occurs for the triple-tube model with periodic boundary conditions. We can now express these non-Lorentz invariant perturbations in terms of the TIM $\times \mathbb{Z}_{3}$ 


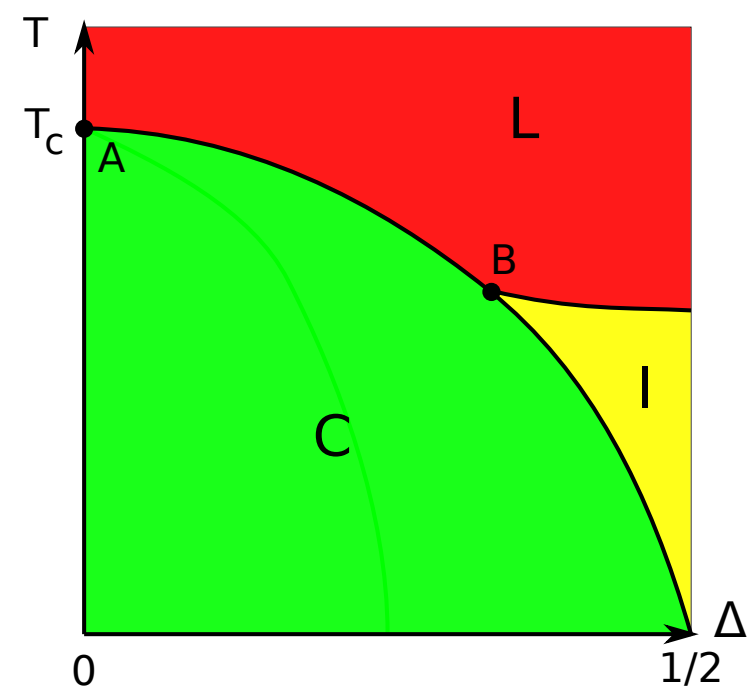

FIG. 4: (color online) Schematic phase diagram of the $2 \mathrm{D} \mathbb{Z}_{3}$ chiral clock model; C, L, and I denote respectively the commensurate, liquid, and incommensurate phase; $T_{c}$ is the critical temperature of the three-state Potts model and $\Delta$ the asymmetry parameter.

degrees of freedom. We find the following identification up to some unimportant normalization factors:

$$
\begin{aligned}
\mathcal{O}_{S= \pm 1}^{(1)} & \sim \mu_{2} \sigma_{\mathrm{TIM}}\left[\Phi_{(2 / 5,7 / 5)}+\Phi_{(7 / 5,2 / 5)}\right] \\
\partial_{x} \Phi_{2} i \xi_{R}^{1} \xi_{L}^{1} & \sim \epsilon_{\mathrm{TIM}}\left[\Phi_{(2 / 5,7 / 5)}+\Phi_{(7 / 5,2 / 5)}\right] .
\end{aligned}
$$

Thus, at energies much smaller than the spectral gap of the Ising and TIM degrees of freedom, the physical properties of triple-tube systems are governed by the effective Hamiltonian (68) with $\delta=\delta^{*}$ (and $\delta_{g}=A\left(-g_{\perp}+g_{d}\right)$ ). The latter Hamiltonian is known to describe the physical properties of the $2 \mathrm{D}$ uniaxial $\mathbb{Z}_{3}$ chiral Potts model, the so-called OstlundHuse model, $\sqrt{36}$ in the vicinity of the three-state Potts critical point. ${ }^{60}$ Interestingly enough, the competition between superfluidity and CDW in triple-tube systems is thus similar to the domain-wall wetting transition in chiral clock model. ${ }^{[6 / 61} \mathrm{We}$ note that a 1D boson chain with constraints also has physical properties described by the $\mathbb{Z}_{3}$ chiral Potts model.62

The Ostlund-Huse model on the square lattice is defined as follows:

$$
\begin{aligned}
\mathcal{H}_{\mathrm{OH}} & =-J \sum_{<i, j>} \cos \left[\frac{2 \pi}{3}\left(n_{i}-n_{j}+\Delta\right)\right] \\
& -J \sum_{<i, k>} \cos \left[\frac{2 \pi}{3}\left(n_{i}-n_{k}\right)\right]
\end{aligned}
$$

where a variable $n_{i}=0,1,2$ is associated with each site $i$ of a square lattice and the sum $\langle i, j\rangle$ (respectively $\langle i, k>$ ) are taken over nearest neighbours in the axial, e.g. $x$, (respectively $y$ ) direction. The Ostlund-Huse model displays a global $\mathbb{Z}_{3}$ symmetry and has been introduced to describe the commensurate-incommensurate transition observed in monolayers absorbed on rectangular substrates ${ }^{36}$ For $\Delta=0$, the model reduces to the ordinary $2 \mathrm{D} \mathbb{Z}_{3}$ clock model, which is equivalent to the three-state Potts model, and enjoys a $S_{3}$ (the permutation group of three objects) global symmetry. Such a model displays a second-order phase transition from a commensurate (or ferromagnetic) phase to a liquid (or paramagnetic) phase in the $\mathbb{Z}_{3}$ parafermionic universality class. The phase diagram of the model with uniaxial anisotropy $\Delta \neq 0$ has proved complicated and very controversial (see Refs 6364 for a review). The main effect of the asymmetry parameter $\Delta$ is to introduce a new incommensurate gapless phase (the so-called floating phase, with central charge $c=1$ ) between the conventional commensurate and liquid phases (see Fig. 4 for a schematic phase diagram). The floating phase is characterized by a modulated order with a wavevector which varies continuously with $T$ and $\Delta$. There is considerable controversy over the possible existence of the Lifshitz point (point B in Fig. 4). To the best of our knowledge, the situation is still not settled. Three different scenari are possible:

(a) There is a Lifshitz point at finite value of $\Delta$ and the Potts critical point (point $\mathrm{A}$ in Fig. 4) extends along the $\mathrm{AB}$ line of Fig. $4 \longdiv { 3 6 \sqrt [ 6 5 ] { 6 6 } }$

(b) The Lifshitz point moves to $\Delta=0$ : there is no direct transition between the commensurate and liquid phase but an intermediate floating phase. ${ }^{67}$.69 There are two phase transitions to reach the liquid phase from the commensurate one: first, a commensurate-incommensurate transition followed by a BKT transition.

(c) There is a Lifshitz point at finite value of $\Delta$ but the $\mathrm{AB}$ line of Fig. 4 is a new critical line with critical exponents different from the three-state Potts universality class. $6170 \mid 71$

In our triple-tube systems, the case (a) corresponds to a $\mathrm{U}(1) \times \mathbb{Z}_{3}$ quantum phase transition between the CDW and superfluid phases, described by the properties 67). In the second case (b), there is no direct transition between these two phases but an intermediate incommensurate gapless phase will then emerge. Adding the contribution of the gapless boson $\Phi_{0}$, this incommensurate gapless phase has central charge $c=1+1=2$. The correlation functions (67), in this floating phase, will be non-universal and incommensurate with a wave-vector $Q \neq 2 \pi \rho_{0}$. We expect the extent of this incommensurate phase to be tiny since $\delta=\delta^{*}$ is small in our approach. Finally, in the last case (c), we still have a direct quantum phase transition between the CDW and superfluid phases. The correlations functions, at the quantum critical point, are still described by Eqs. (67) but with different (unknown) universal exponents.

Unfortunately, the low-energy properties of the effective field theory (68) with $\delta=\delta^{*}$ and $\delta_{g}=0$ are not known and cannot help to resolve the controversy ${ }^{60}$ However, on general grounds, a relevant $S= \pm 1$ conformal spin perturbation usually gives rise to some incommensurate behavior. ${ }^{60 \mid 72}$.74 The second case, i.e. the existence of an intermediate incommensurate gapless phase, thus seems likely. However, relatively recent density-matrix-renormalization group calculations on the Ostlund-Huse model (73) support scenario (c) and no incommensurate gapless phase is found when $\Delta<1 / 4{ }^{71}$ In addition, one might expect a different behavior between the 
two triple-tube systems of Fig. 3 since they do not share the same symmetry. In the equilateral triangle situation, we have a lattice global $S_{3}$ invariance in contrast to the open case (Fig. 3(a)). Since the Ostlund-Huse model does not have this invariance except at $\Delta=0$, it is tempting to conjecture that $\delta=\delta^{*}=0$ in the low-energy effective Hamiltonian (68) for the periodic case. In that case, the quantum phase transition between the superfluid and CDW phases belongs to the U(1) $\times \mathbb{Z}_{3}$ universality class. Unfortunately, within our approach, we cannot directly check that $\delta=\delta^{*}=0$. Indeed, the evaluation of these coupling constants requires the exact knowledge of the non-universal amplitudes on Ising and TIM degrees of freedom in Eq. (72) for the theory (61). In this respect, it would be very interesting in the future, to numerically investigate the zero-temperature phase diagram of lattice triple-tube models of Fig. 3. In particular, it would further shed light on the nature of the quantum phase transition and its relation to the physics of the $2 \mathrm{D} \mathbb{Z}_{3}$ chiral Potts model.

\section{CONCLUDING REMARKS}

In summary, we have investigated the low-energy properties of a system of $N$ 1D dipolar bosons tubes coupled together via intertube hopping and dipole-dipole interactions. Here, we have focused our analysis on the quantum-phase transition for incommensurate filling that reflects the competition between the two different coupling mechanisms using a phenomenological bosonization approach. The transition separates a superfluid phase from a CDW phase when the Luttinger parameter of the bosons fields is $K=1 / 2$. This value can be reached here thanks to the long-range nature of the dipole-dipole interaction. In stark contrast to a single-tube case where the transition is a simple cross-over, the main effect of coupling the 1D tubes through hopping and density interactions is to give rise to a genuine quantum-phase transition.

Using the powerful machinery of the CFT approach, we have determined the main long-wavelength properties of this transition which turns out to be very exotic. In particular, we have revealed that the quantum phase transition is described by the $\mathrm{SU}(2)_{N} \mathrm{CFT}$, or equivalently to the $\mathrm{U}(1) \times \mathbb{Z}_{N} \mathrm{CFT}$, with a fractional central charge $c=3 N /(N+2)$. The $\mathbb{Z}_{N}$ critical degrees of freedom are highly nontrivial and nonlocal with respect to the original atoms or polar molecules of the model. The quantum critical point can be attained by a finetuning of the coupling constants that seem reasonably realistic in the context of cold polar molecules. In this respect, this work opens the possibility to investigate the exotic physics of $\mathbb{Z}_{N}$ parafermions in the context of ultracold quantum bosonic gases.

In the $N=2$ case, we have investigated in details the main characteristics of the quantum critical point which was shown to belong to the $\mathrm{SU}(2)_{2}$ universality class with central charge $c=3 / 2$. The triple-tube case $(N=3)$ is particularly promising since the quantum phase transition is governed by $U(1) \times \mathbb{Z}_{3}$ degrees of freedom. In particular, within our low-energy approach, we have connected this problem to the physics of the $2 \mathrm{D} \mathbb{Z}_{3}$ chiral Potts model. In this respect, it will be important to carry out a thorough large-scale numerical analysis for mapping out the zero-temperature phase diagram of the triple-tube systems by adding an optical lattice in the $x$-direction. The commensurate case is also interesting and will be investigated elsewhere. We hope that ongoing experimental research on polar molecules will allow to probe the quantum phase transition discussed in this paper.

\section{Acknowledgements}

We would like to thank A. A. Nersesyan for encouragement and his interest in the work. We are also grateful to E. Orignac and N. Zinner for hepful discussions.

\section{Appendix A: Free-field representations}

In this Appendix, we present some important technical details on the $\mathrm{SU}(2)_{N}$ quantum critical properties of the effective Hamiltonian (24), which controls the quantum phase transition of the coupled tubes system.

\section{Free-field representation of $\mathrm{SU}(2)_{N} \mathrm{CFT}$}

As stated in Sec. II B, for $g_{\perp}=g_{d}$ and $K=1 / 2$, the Hamiltonian is given by Eq. 27 and displays an enlarged $\mathrm{SU}(2)_{L} \times \mathrm{SU}(2)_{R}$ global symmetry. Let us consider its noninteracting part $\mathcal{H}_{0}^{*}$, where "*" denotes the $g_{\perp}=g_{d}$ point. Each boson $\varphi_{n}$ describes an $\mathrm{SU}(2)_{1}$ CFT with central charge $c=1$, generated by the left and right $\mathrm{SU}(2)_{1}$ current:

$$
\begin{aligned}
j_{n R, L}^{\dagger} & =\frac{1}{2 \pi}: \exp \left(\mp i \sqrt{8 \pi} \varphi_{n R, L}\right): \\
j_{n R, L}^{z} & =\frac{1}{\sqrt{2 \pi}} \partial_{x} \varphi_{n R, L}
\end{aligned}
$$

where : $A$ : is the standard normal ordering of a bosonic operator $A$. In this work, the chiral bosonic fields $\varphi_{n R(L)}$ are defined as:

$$
\begin{aligned}
& \varphi_{n}=\varphi_{n L}+\varphi_{n R} \\
& \vartheta_{n}=\varphi_{n L}-\varphi_{n R}
\end{aligned}
$$

and we are working with the prescription: $\left[\varphi_{n R}, \varphi_{m L}\right]=$ $i \delta_{n m} / 4$. The bosonic fields are normalized by the following operator product expansion (OPE):

$$
\begin{aligned}
\varphi_{n L}(z) \varphi_{m L}(\omega) & \sim-\frac{\delta_{n m}}{4 \pi} \ln (z-\omega), \\
\varphi_{n R}(\bar{z}) \varphi_{m R}(\bar{\omega}) & \sim-\frac{\delta_{n m}}{4 \pi} \ln (\bar{z}-\bar{\omega}),
\end{aligned}
$$

with $z=v \tau+i x, \bar{z}=v \tau-i x$, and $\tau$ is the imaginary time.

Eqs. A1 give a free-boson representation of the $\mathrm{SU}(2)_{1}$ currents that satisfy the so-called $\mathrm{SU}(2)_{1}$ Kac-Moody algebra 
defined by the OPE $! \frac{15}{15}$

$$
j_{n L}^{\alpha}(z) j_{n L}^{\beta}(\omega) \sim \frac{\delta^{\alpha \beta}}{8 \pi^{2}(z-\omega)^{2}}+\frac{i \epsilon^{\alpha \beta \gamma} j_{n L}^{\gamma}(\omega)}{2 \pi(z-\omega)},
$$

with $\alpha, \beta, \gamma=x, y, z$ and a similar result for the right current. Indeed, using Eq. A3 and OPEs involving vertex operators: 15

$$
\begin{aligned}
& : e^{i a \varphi_{n L}}:(z): e^{i b \varphi_{n L}}:(\omega) \\
& \sim(z-\omega)^{a b / 4 \pi}: e^{i a \varphi_{n L}(z)+i b \varphi_{n L}(\omega)}: \\
& \partial_{x} \varphi_{n L}(z): e^{i a \varphi_{n L}}:(\omega) \sim \frac{a}{4 \pi(z-\omega)}: e^{i a \varphi_{n L}}:(\omega),
\end{aligned}
$$

one shows that:

$$
\begin{aligned}
j_{n L}^{ \pm}(z) j_{n L}^{\mp}(\omega) & \sim \frac{1}{4 \pi^{2}(z-\omega)^{2}}: e^{ \pm i \sqrt{8 \pi}\left(\varphi_{n L}(z)-\varphi_{n L}(\omega)\right)}: \\
& \sim \frac{1}{4 \pi^{2}(z-\omega)^{2}} \pm \frac{1}{\pi(z-\omega)} j_{n L}^{z}(\omega), \\
j_{n L}^{ \pm}(z) j_{n L}^{z}(\omega) & \sim \frac{1}{2 \pi \sqrt{2 \pi}} \frac{\mp \sqrt{8 \pi}}{4 \pi(z-\omega)}: e^{ \pm i \sqrt{8 \pi} \varphi_{n L}}:(\omega), \\
& \sim \frac{\mp 1}{2 \pi(z-\omega)} j_{n L}^{ \pm}(\omega), \\
j_{n L}^{z}(z) j_{n L}^{z}(\omega) & \sim \frac{1}{8 \pi^{2}(z-\omega)^{2}} .
\end{aligned}
$$

This is nothing else but the algebra (A4).

As any CFT, the $\mathrm{SU}(2)_{1}$ CFT described by the bosonic fields $\varphi_{n R(L)}$ possesses a stress-energy tensor. Its left - i.e. holomorphic - component is given by:

$$
T_{n}=\frac{4 \pi^{2}}{3}: \mathbf{j}_{n L} \cdot \mathbf{j}_{n L}:
$$

and satisfies the defining relation of a stress-energy tensor of a CFT: 15

$$
T(z) T(\omega) \sim \frac{c / 2}{(z-\omega)^{4}}+\frac{2 T(\omega)}{(z-\omega)^{2}}+\frac{\partial T(\omega)}{z-\omega},
$$

where $\partial=\partial / \partial z$, and $c$ is the central charge for the CFT in question; for the $\mathrm{SU}(2)_{1} \mathrm{CFT}, c=1$ as already mentioned before. Using the representation A1, the $\mathrm{SU}(2)_{1}$ stress-energy tensor can be directly expressed in terms of the boson field:

$$
T_{n}=2 \pi:\left(\partial_{x} \varphi_{n L}\right)^{2}:
$$

it is straightforward to check that the definition $\mathrm{A} 8 \mathrm{~B}$ is indeed reproduced.

The non-interacting part of the Hamiltonian (27), $\mathcal{H}_{0}^{*}$, can be written in terms of the $N \mathrm{SU}(2)_{1}$ currents $\mathrm{A} 1$ :

$$
\mathcal{H}_{0}^{*}=\sum_{n=1}^{N} \int d x \frac{2 \pi v}{3}\left(: \mathbf{j}_{n L} \cdot \mathbf{j}_{n L}:+: \mathbf{j}_{n R} \cdot \mathbf{j}_{n R}:\right) \text {, }
$$

and its underlying CFT is thus $\mathrm{SU}(2)_{1} \times \mathrm{SU}(2)_{1} \times \cdots \times \mathrm{SU}(2)_{1}$ with central charge $N$. If we now combine the $N \mathrm{SU}(2)_{1}$ currents together:

$$
\begin{aligned}
I_{R, L}^{\dagger} & =\frac{1}{2 \pi} \sum_{n=1}^{N}: \exp \left(\mp i \sqrt{8 \pi} \varphi_{n R, L}\right):, \\
I_{R, L}^{z} & =\frac{1}{\sqrt{2 \pi}} \sum_{n=1}^{N} \partial_{x} \varphi_{n R, L},
\end{aligned}
$$

the resulting object is an $\mathrm{SU}(2)_{N}$ current. Indeed, one can show, similarly to the $\mathrm{SU}(2)_{1}$ case, that it satisfies the $\mathrm{SU}(2)_{N}$ Kac-Moody algebra:

$$
I_{L}^{\alpha}(z) I_{L}^{\beta}(\omega) \sim \frac{N \delta^{\alpha \beta}}{8 \pi^{2}(z-\omega)^{2}}+\frac{i \epsilon^{\alpha \beta \gamma} I_{L}^{\gamma}(\omega)}{2 \pi(z-\omega)} .
$$

The holomorphic part of the stress-energy tensor of the $\mathrm{SU}(2)_{N}$ CFT is defined by:

$$
T_{\mathrm{SU}(2)_{N}}=\frac{4 \pi^{2}}{N+2}: \mathbf{I}_{L} \cdot \mathbf{I}_{L}:
$$

It satisfies the relation $\mathrm{A} 8$ with a central charge $c_{\mathrm{SU}(2)_{N}}=$ $3 N /(N+2)$. Using the identification (A11), we find a freefield representation of the $\mathrm{SU}(2)_{N}$ stress-energy tensor in terms of the bosonic fields $\varphi_{n L}$ :

$$
\begin{aligned}
T_{\mathrm{SU}(2) N} & =\frac{6 \pi}{N+2} \sum_{n=1}^{N}:\left(\partial_{x} \varphi_{n L}\right)^{2}: \\
& +\frac{1}{N+2} \sum_{n \neq m=1}^{N}: \cos \left[\sqrt{8 \pi}\left(\varphi_{n L}-\varphi_{m L}\right)\right]: \\
& +\frac{2 \pi}{N+2} \sum_{n \neq m=1}^{N}: \partial_{x} \varphi_{n L} \partial_{x} \varphi_{m L}:
\end{aligned}
$$

\section{Self-dual perturbation and $\mathcal{G}_{N}$ primary field}

In Sec. IIC in order to investigate the critical properties of the model, we have introduced the $\mathcal{G}_{N}$ CFT through the conformal embedding (30):

$$
\mathrm{SU}(2)_{1} \times \mathrm{SU}(2)_{1} \times \cdots \times \mathrm{SU}(2)_{1} \rightarrow \mathrm{SU}(2)_{N} \times \mathcal{G}_{N} .
$$

Its stress-energy tensor is the difference between the $N$ $\mathrm{SU}(2)_{1}$ and the $\mathrm{SU}(2)_{N}$ stress-energy tensors, i.e., in the bosonic description:

$$
\begin{aligned}
T_{\mathcal{G}_{N}} & =\left(\sum_{n=1}^{N} T_{n}\right)-T_{S U(2)_{N}} \\
& =\frac{2 \pi(N-1)}{N+2} \sum_{n}:\left(\partial_{x} \varphi_{n L}\right)^{2}: \\
& -\frac{1}{N+2} \sum_{n \neq m}: \cos \left(\sqrt{8 \pi}\left(\varphi_{n L}-\varphi_{m L}\right)\right): \\
& -\frac{2 \pi}{N+2} \sum_{n \neq m}: \partial_{x} \varphi_{n L} \partial_{x} \varphi_{m L}:
\end{aligned}
$$


Let us show that indeed $T_{\mathcal{G}_{N}}$ satisfies the OPE of stress-energy tensors A8 with a central charge $c_{\mathrm{G}_{N}}=N(N-1) /(N+2)$. Since we already know the OPE that $T_{n}$ and $T_{S U(2)_{N}}$ satisfy (see Eq. (A8)), we only need to compute the OPE between the $N \mathrm{SU}(2)_{1}$ and the $\mathrm{SU}(2)_{N}$ stress-energy tensors. Using the free-field representations A14, A17) and Eq. (A5), one obtains:

$$
\begin{aligned}
T_{\mathrm{SU}(2)_{N}}(z) \sum_{n=1}^{N} T_{n}(\omega) & \sim \frac{3 N}{2(N+2)} \frac{1}{(z-\omega)^{4}}+\frac{2 T_{\mathrm{SU}(2)_{N}}(\omega)}{(z-\omega)^{2}} \\
& +\frac{\partial T_{\mathrm{SU}(2)_{N}}(\omega)}{z-\omega} .
\end{aligned}
$$

The OPE $\sum_{n=1}^{N} T_{n}(z) T_{\mathrm{SU}(2)_{N}}(\omega)$ can be easily deduced from the former by exchanging $z$ and $\omega$, then performing an expansion of $z$ around $\omega$; it has the exact same form. Combining all these OPEs together, we finally have:

$$
\begin{aligned}
& T_{\mathcal{G}_{N}}(z) T_{\mathcal{G}_{N}}(\omega) \\
= & \sum_{n, m=1}^{N} T_{n}(z) T_{m}(\omega)+T_{S U(2)_{N}}(z) T_{S U(2)_{N}}(\omega) \\
& -T_{\mathrm{SU}(2)_{N}}(z) \sum_{n=1}^{N} T_{n}(\omega)-\sum_{n=1}^{N} T_{n}(z) T_{\mathrm{SU}(2)_{N}}(\omega) \\
\sim & \frac{N(N-1)}{2(N+2) z^{4}}+\frac{2 T_{\mathcal{G}_{N}}(\omega)}{(z-\omega)^{2}}+\frac{\partial T_{\mathcal{G}_{N}}(\omega)}{z-\omega},
\end{aligned}
$$

which proves the value of the central charge $c_{\mathrm{G}_{N}}=N(N-$ 1) $/(N+2)$ asserted above.

In the analysis of Sec. II C, we have used some crucial properties of the self-dual term (32) that is responsible for the quantum phase transition in the dipolar bosons tubes. With the definitions above at hand, we now demonstrate these properties. In terms of the bosonic fields, the self-dual term reads:

$$
\begin{array}{r}
\mathcal{V}_{\mathrm{sd}}=\sum_{i=1}^{N-1}: \cos \left(\sqrt{2 \pi}\left(\varphi_{i+1}-\varphi_{i}\right)\right): \\
\quad+: \cos \left(\sqrt{2 \pi}\left(\vartheta_{i+1}-\vartheta_{i}\right)\right): .
\end{array}
$$

Let us check explicitely that it is a singlet under the $\mathrm{SU}(2)_{N}$ CFT:

$$
\vec{I}_{L}(z) \mathcal{V}_{\mathrm{sd}}(\omega, \bar{\omega}) \sim \overrightarrow{0}
$$

and similarly for the right component. First of all, we have, using Eq. A5):

$$
\begin{aligned}
& I_{L}^{x}(z): \cos \sqrt{2 \pi}\left(\varphi_{k+1}-\varphi_{k}\right):(\omega, \bar{\omega}) \\
& \sim \frac{1}{4 \pi} \sum_{i} \frac{-i}{(z-\omega)}\left(\delta^{i, k+1}\left[\widetilde{C}_{k+1} C_{k}-\widetilde{S}_{k+1} S_{k}\right]\right. \\
& \left.+\delta^{i, k}\left[C_{k+1} \widetilde{C}_{k}-S_{k+1} \widetilde{S}_{k}\right]\right)(\omega, \bar{\omega}), \\
& \sim \frac{-i}{4 \pi(z-\omega)}\left(\widetilde{C}_{k+1} C_{k}-\widetilde{S}_{k+1} S_{k}\right. \\
& \left.+C_{k+1} \widetilde{C}_{k}-S_{k+1} \widetilde{S}_{k}\right)(\omega, \bar{\omega}), \\
& I_{L}^{x}(z): \cos \left(\sqrt{2 \pi}\left(\vartheta_{k+1}-\vartheta_{k}\right)\right):(\omega, \bar{\omega}) \\
& \sim \frac{i}{4 \pi(z-\omega)}\left(C_{k+1} \widetilde{C}_{k}-S_{k+1} \widetilde{S}_{k}\right. \\
& \left.+\widetilde{C}_{k+1} C_{k}-\widetilde{S}_{k+1} S_{k}\right)(\omega, \bar{\omega}),
\end{aligned}
$$

where we have adopted the shorthand notation:

$$
: \cos \left(\sqrt{2 \pi} \varphi_{k}\right):=C_{k}, \quad: \cos \left(\sqrt{2 \pi} \vartheta_{k}\right):=\widetilde{C}_{k}
$$

and with similar definitions for the sine functions $S_{k}$ and $\widetilde{S}_{k}$. We conclude thus: $I_{L}^{x}(z) \mathcal{V}_{\mathrm{sd}}(\omega, \bar{\omega}) \sim 0$, and similarly for the $y$ component of the $\mathrm{SU}(2)_{N}$ current $I_{L}^{y}$. For the $z$ component, we have, using Eq. A5:

$$
\begin{aligned}
& I_{L}^{z}(z): \cos \sqrt{2 \pi}\left(\varphi_{k+1}-\varphi_{k}\right):(\omega, \bar{\omega}) \\
& \sim \frac{i}{4 \pi} \sum_{i} \frac{1}{z-\omega}\left(\delta^{i, k+1}: \sin \sqrt{2 \pi}\left(\varphi_{k+1}-\varphi_{k}\right):\right. \\
& \left.\quad-\delta^{i, k}: \sin \sqrt{2 \pi}\left(\varphi_{k+1}-\varphi_{k}\right):\right)(\omega, \bar{\omega})
\end{aligned}
$$

$\sim 0$.

The same also applies for the second term of $\mathcal{V}_{\mathrm{sd}}$ and that finally proves Eq. A21.

The second important property is that $\mathcal{V}_{\mathrm{sd}}$ is a primary field under the $\mathcal{G}_{N}$ CFT, with scaling dimension $\Delta_{\mathcal{V}}=1$ :

$$
T_{\mathcal{G}_{N}}(z) \mathcal{V}_{\mathrm{sd}}(\omega, \bar{\omega}) \sim \frac{\mathcal{V}_{\mathrm{sd}}(\omega, \bar{\omega})}{2(z-\omega)^{2}}+\frac{\partial \mathcal{V}_{\mathrm{sd}}(\omega, \bar{\omega})}{z-\omega}
$$

Let us check that $\mathcal{V}_{\text {sd }}$ displays such property. First, we have just shown that $\overrightarrow{I_{L}}(z) \mathcal{V}_{\text {sd }}(\omega, \bar{\omega}) \sim 0$, so it is straightforward to see that $T_{\mathrm{SU}(2)_{N}}(z) \mathcal{V}_{\mathrm{sd}}(\omega, \bar{\omega}) \sim 0$. We are left with the OPE 
of the $N \mathrm{SU}(2)_{1}$ stress-energy tensors with $\mathcal{V}_{\mathrm{sd}}$ :

$$
\begin{aligned}
& \sum_{n=1}^{N} T_{n}(z): \cos \left[\sqrt{2 \pi}\left(\varphi_{k}-\varphi_{k+1}\right)\right]:(\omega, \bar{\omega}) \\
& \sim \sum_{n=1}^{N} \frac{1}{4} \frac{1}{(z-\omega)^{2}}\left(\delta_{n, k}+\delta_{n, k+1}\right) \\
& \times:\left[C_{k} C_{k+1}+S_{k} S_{k+1}\right]:(\omega, \bar{\omega}) \\
& +\sum_{n=1}^{N} \frac{1}{z-\omega}\left\{\delta_{n, k}\left[\partial\left(C_{k}\right) C_{k+1}+\partial\left(S_{k}\right) S_{k+1}\right]\right. \\
& \left.+\delta_{n, k+1}\left[C_{k} \partial\left(C_{k+1}\right)+S_{k} \partial\left(S_{k+1}\right)\right]\right\}(\omega, \bar{\omega}), \\
& \sim \frac{1}{2} \frac{1}{(z-\omega)^{2}}: \cos \left[\sqrt{2 \pi}\left(\varphi_{k}-\varphi_{k+1}\right)\right]:(\omega, \bar{\omega}) \\
& +\frac{1}{z-\omega} \partial\left(: \cos \left[\sqrt{2 \pi}\left(\varphi_{k}-\varphi_{k+1}\right)\right]:\right)(\omega, \bar{\omega}),
\end{aligned}
$$

and similarly for the second term of $\mathcal{V}_{\mathrm{sd}}$, which proves finally Eq. A25.

\section{Free-field representation of the TIM and $\mathbb{Z}_{3}$ Potts CFTs}

In this section, we give a proof of the non-trivial identification (56) which occurs in triple-tube systems.

It is first important to observe that the free massless bosonic field $\Phi_{2}$ has a very special compactification radius $R_{2}=$ $\sqrt{3 / \pi}$ in the classification of the CFT with central charge $c=1$. At this radius, it displays a CFT with an extended symmetry: a $N=2$ (respectively $N=1$ ) superconformal field theory (SCFT) when the bosonic field $\Phi_{2}$ is compactified along a circle (respectively an orbifold $\Phi_{2} \sim-\Phi_{2}$ ) with radius $R_{2}{ }^{[75}$ Forgetting the contribution of the Majorana fermion $\xi_{L}^{2}$, the conformal symmetry of the non-interacting limit of model (55) is Ising $\times[c=1$ SCFT], with central charge $c=3 / 2$. The latter CFT can also be described in terms of the product of TIM $\times \mathbb{Z}_{3}$ Potts CFTs with central charge $c=7 / 10+4 / 5=3 / 2$. The precise conformal embedding has been derived by the authors of Ref 48 .

$$
\mathbb{Z}_{2} \times(c=1 N=1 \mathrm{SCFT})=P\left[\mathcal{M}_{4} \times \mathcal{M}_{5}\right]
$$

where the projection $P$ restricts the primary operators of $\mathcal{M}_{4} \times \mathcal{M}_{5}$ to the subset: $\left\{\Phi_{r, s}^{(4)} \Phi_{s, q}^{(5)}\right\}, \Phi_{r, s}^{(p)}(1 \leq r \leq p-1,1 \leq$ $s \leq p)$ being the primary operator of $\mathcal{M}_{p}$ CFT.

As is well-known, the (left) stress-energy tensor of the $\mathbb{Z}_{2}$ (or Ising) CFT expresses directly in terms of the Majorana fermion $\xi_{L}^{1}: T_{I}=-\pi: \xi_{L}^{1} \partial \xi_{L}^{1}:$. It is indeed straightforward to check that $T_{I}$ satisfies the defining relation (A8) of a stressenergy tensor with $c=1 / 2$. The left Majorana $\xi_{L}^{1}$ is a primary field with holomorphic weight $h=1 / 2$ :

$$
\begin{aligned}
& T_{I}(z) \xi_{L}^{1}(0) \sim \frac{\xi_{L}^{1}(0)}{2 z^{2}}+\frac{\partial \xi_{L}^{1}(0)}{z} \\
& \xi_{L}^{1}(z) \xi_{L}^{1}(0) \sim \frac{1}{2 \pi z}+\frac{z}{\pi} T_{I}(0)+\frac{z^{2}}{2 \pi} \partial T_{I}(0) .(
\end{aligned}
$$

For the $N=1$ SCFT with central charge $c=1$, the stressenergy tensor is simply given by Eq. (A9): $T_{0}=-2 \pi$ : $\left(\partial \Phi_{2 L}\right)^{2}:$. On top of this bosonic tensor, the $N=1$ SCFT is characterized by the existence of a fermionic current $G$ with holomorphic weight $h=3 / 2: 76$

$$
\begin{aligned}
T_{0}(z) G(0) & \sim \frac{3 G(0)}{2 z^{2}}+\frac{\partial G(0)}{z} \\
G(z) G(0) & \sim \frac{1}{z^{3}}+\frac{3 T_{0}(0)}{z}+\frac{3 \partial T_{0}(0)}{2} .
\end{aligned}
$$

In the particular case of the $N=1$ SCFT with central charge $c=1$, the current $G$ has a simple free-field representation in terms of the free-massless boson $\Phi_{2 L}: \frac{.75}{}$

$$
G=\sqrt{2}: \cos \left(\sqrt{12 \pi} \Phi_{2 L}\right):
$$

It is indeed easy to check, using Eq. A5, that the OPEs A29) are reproduced from this identification.

From all these definitions, we find a new free-field representation of the left stress-energy tensors of the TIM and $\mathbb{Z}_{3}$ Potts CFTs in terms of a Majorana fermion and a freemassless boson:

$$
\begin{aligned}
T_{\mathrm{TIM}} & =\frac{1}{5} T_{I}+\frac{3}{5} T_{0}-\frac{4 \sqrt{\pi}}{5} \xi_{L}^{1}: \cos \left(\sqrt{12 \pi} \Phi_{2 L}\right): \\
T_{\mathbb{Z}_{3}} & =\frac{4}{5} T_{I}+\frac{2}{5} T_{0}+\frac{4 \sqrt{\pi}}{5} \xi_{L}^{1}: \cos \left(\sqrt{12 \pi} \Phi_{2 L}\right):
\end{aligned}
$$

where $T_{\mathrm{TIM}}$ and $T_{\mathbb{Z}_{3}}$ denote respectively the stress-energy tensor of the TIM and $\mathbb{Z}_{3}$ Potts CFTs. Using the results A28, A29, one can indeed show that $T_{\text {TIM }}$ (respectively $T_{\mathbb{Z}_{3}}$ ) satisfies the definition A8 with $c=7 / 10$ (respectively $c=4 / 5$ ), together with the decoupling: $T_{\mathrm{TIM}}(z) T_{\mathbb{Z}_{3}}(0) \sim 0$. In addition, we note that we have $T_{\mathrm{TIM}}+T_{\mathbb{Z}_{3}}=T_{I}+T_{0}$ in full agreement with the existence of the conformal embedding (A27).

We are now in position with the free-field representation A31) to show the identification 56. In this respect, let us introduce the following operators:

$$
\begin{aligned}
& \mathcal{O}_{1}=\mu_{1}: \cos \left(\sqrt{3 \pi} \Phi_{2}\right):+\sigma_{1}: \cos \left(\sqrt{3 \pi} \Theta_{2}\right): \\
& \mathcal{O}_{2}=\mu_{1}: \cos \left(\sqrt{3 \pi} \Phi_{2}\right):-\sigma_{1}: \cos \left(\sqrt{3 \pi} \Theta_{2}\right):
\end{aligned}
$$

Let us first show that $\mathcal{O}_{1}$ is a singlet under the $\mathbb{Z}_{3}$ Potts CFT and a primary operator of the TIM CFT with holomorphic weight $h=7 / 16$. To this end, we need the following OPEs for a massless boson field that can be obtained from Eq. (A5):

$$
\begin{aligned}
& : \cos \left(\sqrt{12 \pi} \Phi_{2 L}\right):(z): \cos \left(\sqrt{3 \pi} \Phi_{2}\right):(0,0) \sim \\
& \frac{e^{-i 3 \pi / 4}}{2 z^{3 / 2}}\left[: \cos \left(\sqrt{3 \pi} \Theta_{2}\right):+2 z: \partial \cos \left(\sqrt{3 \pi} \Theta_{2}\right):\right](0,0) \\
& : \cos \left(\sqrt{12 \pi} \Phi_{2 L}\right):(z): \cos \left(\sqrt{3 \pi} \Theta_{2}\right):(0,0) \sim \\
& \frac{e^{i 3 \pi / 4}}{2 z^{3 / 2}}\left[: \cos \left(\sqrt{3 \pi} \Phi_{2}\right):+2 z: \partial \cos \left(\sqrt{3 \pi} \Phi_{2}\right):\right](0,0)
\end{aligned}
$$


The following Ising OPEs, which can be found in Ref 77, are also needed:

$$
\begin{aligned}
\xi_{L}^{1}(z) \sigma_{1}(0,0) & \sim \frac{e^{i \pi / 4}}{2 \sqrt{\pi} z^{1 / 2}}\left[\mu_{1}+4 z \partial \mu_{1}\right](0,0) \\
\xi_{L}^{1}(z) \mu_{1}(0,0) & \sim \frac{e^{-i \pi / 4}}{2 \sqrt{\pi} z^{1 / 2}}\left[\sigma_{1}+4 z \partial \sigma_{1}\right](0,0) .
\end{aligned}
$$

With all these identifications, it becomes straightforward to obtain the following result directly from Eq. (A31):

$$
\begin{aligned}
T_{\mathrm{TIM}}(z) \mathcal{O}_{1}(0,0) & \sim \frac{7 \mathcal{O}_{1}(0,0)}{16 z^{2}}+\frac{\partial \mathcal{O}_{1}(0,0)}{z} \\
T_{\mathbb{Z}_{3}}(z) \mathcal{O}_{1}(0,0) & \sim 0
\end{aligned}
$$

which states that $\mathcal{O}_{1}$ is indeed a primary operator of the TIM CFT with scaling dimension $7 / 8$. It is thus proportional to the subleading magnetization $\sigma_{\mathrm{TIM}}^{\prime}$. Using the correlation 49 , one can fix the normalization factor by evaluating the twopoint function of $\mathcal{O}_{1}$ :

$$
\begin{aligned}
\left\langle\mathcal{O}_{1}(z, \bar{z}) \mathcal{O}_{1}(0,0)\right\rangle & \sim|z|^{-7 / 4} \\
& =\left\langle\sigma_{\mathrm{TIM}}^{\prime}(z, \bar{z}) \sigma_{\mathrm{TIM}}^{\prime}(0,0)\right\rangle
\end{aligned}
$$

so that we deduce $\mathcal{O}_{1} \sim \sigma_{\text {TIM }}^{\prime}$. Finally, from its definition A32, it is easy to check that $\mathcal{O}_{2}$ is a primary operator of the $\mathcal{M}_{4} \times \mathcal{M}_{5}$ CFT with scaling dimension $7 / 8$ :

$$
\begin{aligned}
\left(T_{\mathrm{TIM}}+T_{\mathbb{Z}_{3}}\right)(z) \mathcal{O}_{2}(0,0) & =\left(T_{I}+T_{0}\right)(z) \mathcal{O}_{2}(0,0) \quad(\mathrm{A} 37) \\
& \sim \frac{7 \mathcal{O}_{2}(0,0)}{16 z^{2}}+\frac{\partial \mathcal{O}_{2}(0,0)}{z} .
\end{aligned}
$$

The $P\left[\mathcal{M}_{4} \times \mathcal{M}_{5}\right]$ CFT contains only two primary operators with scaling dimension $7 / 8$, i.e., $\sigma_{\mathrm{TIM}}^{\prime}$ and $\sigma_{\mathrm{TIM}} \epsilon_{\mathbb{Z}_{3}}$. We thus find that $\mathcal{O}_{2} \sim \sigma_{\mathrm{TIM}} \epsilon_{\mathbb{Z}_{3}}$ since $\mathcal{O}_{1} \sim \sigma_{\mathrm{TIM}}^{\prime}$.
${ }^{1}$ I. Bloch, J. Dalibard, and W. Zwerger, Rev. Mod. Phys. 80, 885 (2008).

2 T. Lahaye, C. Menotti, L. Santos, M. Lewenstein, and T. Pfau, Rep. Prog. Phys. 72, 126401 (2009).

${ }^{3}$ K.-K. Ni, S. Ospelkaus, M. H. G. de Miranda, A. Pe'er, B. Neyenhuis, J. J. Zirbel, S. Kotochigova, P. S. Julienne, D. S. Jin, and J. Ye, Science 322, 231 (2008); S. Ospelkaus, K.-K. Ni, M. H. G. de Miranda, B. Neyenhuis, D. Wang, S. Kotochigova, P. S. Julienne, D. S. Jin, and J. Ye, Faraday Discuss. 142, 351 (2009).

${ }^{4}$ K. Aikawa, D. Akamatsu, M. Hayashi, K. Oasa, J. Kobayashi, P. Naidon, T. Kishimoto, M. Ueda, and S. Inouye, Phys. Rev. Lett. 105, 203001 (2010)

5 A. Micheli, G. Pupillo, H. P. Buchler, and P. Zoller, Phys. Rev. A 76, 043604 (2007).

${ }^{6}$ A. V. Gorshkov, P. Rabl, G. Pupillo, A. Micheli, P. Zoller, M. D. Lukin, and H. P. Buchler, Phys. Rev. Lett. 101, 073201 (2008).

7 J. Levinsen, N. R. Cooper, and G. V. Shlyapnikov, Phys. Rev. A 84, 013603 (2011); N. R. Cooper and G. V. Shlyapnikov, Phys. Rev. Lett. 103, 155302 (2009).

${ }^{8}$ S. T. Carr, J. Quintanilla, and J. J. Betouras, Phys. Rev. B 82, 045110 (2010); J. Quintanilla, S. T. Carr, and J. J. Betouras, Phys. Rev. A 79, 031601 (2009).

${ }^{9}$ B. M. Fregoso, K. Sun, E. Fradkin, and B. L. Lev, New J. Phys. 11, 103003 (2009).

${ }^{10}$ A. V. Gorshkov, S. R. Manmana, G. Chen, E. Demler, M. D. Lukin, and A. M. Rey, Phys. Rev. A 84, 033619 (2011); A. V. Gorshkov, S. R. Manmana, G. Chen, J. Ye, E. Demler, M. D. Lukin, and A. Maria Rey, Phys. Rev. Lett. 107, 115301 (2011).

11 F. D. M. Haldane, Phys. Rev. Lett. 47, 1840 (1981).

12 T. Giamarchi, Quantum Physics in One Dimension (Clarendon press, Oxford, UK, 2004).

13 M. A. Cazalilla, J. Phys. B 37, S1 (2004).

${ }^{14}$ M. A. Cazalilla, R. Citro, T. Giamarchi, E. Orignac, and M. Rigol, Rev. Mod. Phys. 83, 1405 (2011).

15 P. Di Francesco, P. Mathieu, and D. Sénéchal, Conformal Field Theory (Springer, Berlin, 1997).

16 A. O. Gogolin, A. A. Nersesyan, and A. M. Tsvelik, Bosonization and Strongly Correlated Systems (Cambridge University Press, Cambridge, England, 1998)

17 M. W. J. Romans, R. A. Duine, S. Sachdev, and H. T. C. Stoof,
Phys. Rev. Lett. 93, 020405 (2004).

${ }^{18}$ E. Berg, E. G. Dalla Torre, T. Giamarchi, and E. Altman, Phys. Rev. B 77, 245119 (2008).

19 S. Diehl, M. Baranov, A. J. Daley, and P. Zoller, Phys. Rev. Lett. 104, 165301 (2010).

${ }^{20}$ M. J. Bhaseen, S. Ejima, M. Hohenadler, A. O. Silver, F. H. L. Essler, H. Fehske, and B. D. Simons, Phys. Rev. A 84, 023635 (2011); S. Ejima, M. J. Bhaseen, M. Hohenadler, F. H. L. Essler, H. Fehske, and B. D. Simons, Phys. Rev. Lett. 106, 015303 (2011); M. Hohenadler, A. O. Silver, M. J. Bhaseen, and B. D. Simons, Phys. Rev. A 82, 013639 (2010).

21 J. Ruhman, E. G. Dalla Torre, S. D. Huber, and E. Altman, arXiv:1111.0778.

${ }^{22}$ V. G. Knizhnik and A. B. Zamolodchikov, Nucl. Phys. B 247, 83 (1984).

23 A. B. Zamolodchikov and V. A. Fateev, Sov. Phys. JETP 62, 215 (1985).

${ }^{24}$ D. Gepner and Z Qiu, Nucl. Phys. B 285, 423 (1987).

${ }^{25}$ N. Read and E. H. Rezayi, Phys. Rev. B 59, 8084 (1999).

${ }^{26}$ J. M. Fellows and S. T. Carr, Phys. Rev. A 84, 051602(R) (2011).

27 A. Arguelles and L. Santos, Phys. Rev. A 75, 053613 (2007).

${ }^{28}$ C. Kollath, J. S. Meyer, and T. Giamarchi, Phys. Rev. Lett. 100, 130403 (2008)

${ }^{29}$ C.-M. Chang, W.-C. Shen, C.-Y. Lai, P. Chen, and D.-W. Wang, Phys. Rev. A 79, 053630 (2009); Y.-P. Huang and D.-W. Wang, Phys. Rev. A 80, 053610 (2009).

${ }^{30}$ M. Dalmonte, G. Pupillo, and P. Zoller, Phys. Rev. Lett. 105, 140401 (2010); M. Dalmonte, P. Zoller, and G. Pupillo, Phys. Rev. Lett. 107, 163202 (2011).

${ }^{31}$ N. T. Zinner, B. Wunsch, I. B. Mekhov, S. J. Huang, D.-W. Wang, and E. Demler, Phys. Rev. A 84, 063606 (2011); B. Wunsch, N. T. Zinner, I. B. Mekhov, S.-J. Huang, D.-W. Wang, and E. Demler, Phys. Rev. Lett. 107, 073201 (2011).

32 S. Giovanazzi, A. Gorlitz, and T. Pfau, Phys. Rev. Lett. 89, 130401 (2002).

33 J. Sebby-Strabley, M. Anderlini, P. S. Jessen, and J. V. Porto, Phys. Rev. A 73, 033605 (2006).

${ }^{34}$ A. F. Ho, M. A. Cazalilla, and T. Giamarchi, Phys. Rev. Lett. 92, 130405 (2004); M. A. Cazalilla, A. Iucci, and T. Giamarchi, Phys. Rev. A 75, 051603 (2007). 
35 R. Citro, E. Orignac, S. De Palo, and M. L. Chiofalo, Phys. Rev. A 75, 051602(R) (2007); R. Citro, S. De Palo, E. Orignac, P. Pedri, and M. L. Chiofalo, New J. Phys. 10, 045011 (2008).

36 S. Ostlund, Phys. Rev. B 24, 398 (1981); D. A. Huse, Phys. Rev. B 24, 5180 (1981).

${ }^{37}$ M. Knap, E. Berg, M. Ganahl, and E. Demler, arXiv: 1112.5662; M. Bauer and M. Parish, arXiv: 1202.4151.

${ }^{38}$ S. T. Carr and A. M. Tsvelik, Phys. Rev. B 65, 195121 (2002); F. H. L. Essler and A. M. Tsvelik, Phys. Rev. B 65, 115117 (2002).

39 A. Jaefari, S. Lal, and E. Fradkin, Phys. Rev. B 82, 144531 (2010); E. Arrigoni, E. Fradkin, and S. A. Kivelson, Phys. Rev. B 69, 214519 (2004).

${ }^{40}$ P. Lecheminant, Phys. Lett. B 648, 323 (2007).

41 J. C. Y. Teo and C. L. Kane, arXiv: 1111.2617.

${ }^{42}$ P. Lecheminant, E. Boulat, and P. Azaria, Phys. Rev. Lett. 95, 240402 (2005); P. Lecheminant, P. Azaria, and E. Boulat, Nucl. Phys. B 798, 443 (2008).

${ }^{43}$ C. Wu, Phys. Rev. Lett. 95, 266404 (2005).

${ }^{44}$ S. Capponi, G. Roux, P. Lecheminant, P. Azaria, E. Boulat, and S. R. White, Phys. Rev. A 77, 013624 (2008); G. Roux, S. Capponi, P. Lecheminant, and P. Azaria, Eur. Phys. J. B 68, 293 (2009).

45 P. Azaria, S. Capponi, and P. Lecheminant, Phys. Rev. A 80, 041604 (2009).

46 A. Kantian, M. Dalmonte, S. Diehl, W. Hofstetter, P. Zoller, and A. J. Daley, Phys. Rev. Lett. 103, 240401 (2009).

47 R. A. Molina, J. Dukelsky, and P. Schmitteckert, Phys. Rev. A 80, 013616 (2009).

48 C. Crnkovic, R. Paunov, G. M. Sotkov, and M. Stanishkov, Nucl. Phys. B 336, 637 (1990).

49 V. A. Fateev, Int. J. Mod. Phys. A 6, 2109 (1991).

${ }^{50}$ P. Dorey, R. Tateo, and K. E. Thompson, Nucl. Phys. B 470, 317 (1996).

51 E. Orignac and T. Giamarchi, Phys. Rev. B 57, 11713 (1998).

52 M. C. Ogilvie, Ann. Phys. 136, 273 (1981); P. Lecheminant, A. O. Gogolin, and A. A. Nersesyan, Nucl. Phys. B 639, 502 (2002).

53 J. B. Zuber and C. Itzykson, Phys. Rev. D 15, 2875 (1977); B. Schroer and T. T. Truong, Nucl. Phys. B 144, 80 (1978).

${ }^{54}$ D. Boyanovsky, Phys. Rev. B 39, 6744 (1989).

55 D. G. Shelton, A. A. Nersesyan, and A. M. Tsvelik, Phys. Rev. B 53, 8521 (1996).
56 D. Allen and D. Senechal, Phys. Rev. B 55, 299 (1997).

57 M. Fabrizio, A. O. Gogolin, and A. A. Nersesyan, Nucl. Phys. B 580, 647 (2000).

58 M. Lassig, G. Mussardo, and J. L. Cardy, Nucl. Phys. B 348, 591 (1991).

59 P. Lecheminant and E. Orignac, Phys. Rev. B 69, 174409 (2004).

${ }^{60}$ J. L. Cardy, Nucl. Phys. B 389, 577 (1993).

${ }^{61}$ D. A. Huse and M. E. Fisher, Phys. Rev. B 29, 239 (1984); D. A. Huse and M. E. Fisher, Phys. Rev. Lett. 49, 793 (1982).

${ }^{62}$ P. Fendley, K. Sengupta, and S. Sachdev, Phys. Rev. B 69, 075106 (2004).

${ }^{63}$ M. den Nijs, in Phase Transitions and Critical Phenomena, edited by C. Domb and J. Lebowitz, Vol. 12 (Academic Press, New York, 1988).

${ }^{64}$ H. Au-Yang and J. H. H. Perk, Physica A 228, 78 (1996).

65 S. Howes, L. P. Kadanoff, and M. Den Nijs, Nucl. Phys. B 215, 169 (1983).

${ }^{66}$ M. D. Duxbury, J. Yeomans, and P. D. Beale, J. Phys. A: Math. Gen. 17, L179 (1984); W. Selke and J. Yeomans, Z. Phys. B 46, 311 (1982); J. Yeomans and B. Derrida, J. Phys. A: Math. Gen. 18, 2343 (1985).

${ }^{67}$ F. D. M. Haldane, P. Bak, and T. Bohr, Phys. Rev. B 28, 2743 (1983).

${ }^{68}$ H. J. Schulz, Phys. Rev. B 28, 2746 (1983).

${ }^{69}$ G. von Gehlen and V. Rittenberg, Nucl. Phys. B 230, 455 (1984).

${ }^{70}$ P. Centen, V. Rittenberg, and M. Marcu, Nucl. Phys. B 205, 585 (1982).

${ }^{71}$ H. Sato and K. Sasaki, J. Phys. Soc. Jpn. 69, 1050 (2000).

72 A. A. Nersesyan, A. O. Gogolin, and F. H. L. Essler, Phys. Rev. Lett. 81, 910 (1998).

73 D. Allen, P. Azaria, and P. Lecheminant, J. Phys. A: Math. Gen. 34, L305 (2001).

74 A. M. Tsvelik, Nucl. Phys. B 612, 479 (2001).

75 G. Waterson, Phys. Lett. B 171, 77 (1986); S.-K. Yang, and H. B. Zheng, Nucl. Phys. B 285, 410 (1987).

76 D. Friedan, Z. Qiu, and S. Shenker, Phys. Lett. B 151, 37 (1985).

77 R. Chatterjee and A. B. Zamolodchikov, Mod. Phys. Lett. A 9, 2227 (1994). 